\title{
Differing Effects of Standard and Harsh Nucleic Acid Extraction Procedures on Diagnostic Helminth Real-Time PCRs Applied to Human Stool Samples
}

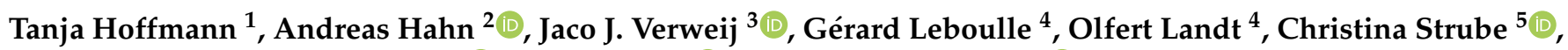
Simone Kann ${ }^{6}$, Denise Dekker ${ }^{7}\left(\mathbb{D}\right.$, Jürgen May ${ }^{7}{ }^{1}$, Hagen Frickmann ${ }^{1,2,+}{ }^{-1}$ and Ulrike Loderstädt ${ }^{8, *},+$

check for updates

Citation: Hoffmann, T.; Hahn, A.; Verweij, J.J.; Leboulle, G.; Landt, O.; Strube, C.; Kann, S.; Dekker, D.; May, J.; Frickmann, H.; et al. Differing Effects of Standard and Harsh Nucleic Acid Extraction Procedures on Diagnostic Helminth Real-Time PCRs Applied to Human Stool Samples. Pathogens 2021, 10, 188. https: / /doi.org/10.3390/ pathogens 10020188

Academic Editors: Roberto Amerigo Papini and Hans-Peter Fuehrer

Received: 8 January 2021

Accepted: 5 February 2021

Published: 9 February 2021

Publisher's Note: MDPI stays neutral with regard to jurisdictional claims in published maps and institutional affiliations.

Copyright: (c) 2021 by the authors. Licensee MDPI, Basel, Switzerland. This article is an open access article distributed under the terms and conditions of the Creative Commons Attribution (CC BY) license (https:/ / creativecommons.org/licenses/by/ $4.0 /)$.
1 Department of Microbiology and Hospital Hygiene, Bundeswehr Hospital Hamburg, 20359 Hamburg, Germany; tanja1hoffmann@bundeswehr.org (T.H.); frickmann@bnitm.de or hagen.frickmann@med.uni-rostock.de (H.F.)

2 Institute for Medical Microbiology, Virology and Hygiene, University Medicine Rostock, 18057 Rostock, Germany; hahn.andreas@me.com

3 Laboratory for Medical Microbiology and Immunology, Elisabeth Tweesteden Hospital, 5042 AD Tilburg, The Netherlands; j.verweij@etz.nl

4 TIB MOLBIOL, 12103 Berlin, Germany; gleboulle@tib-molbiol.de (G.L.); olandt@tib-molbiol.de (O.L.)

5 Institute for Parasitology, Centre for Infection Medicine, University of Veterinary Medicine Hannover, 30559 Hannover, Germany; christina.strube@tiho-hannover.de

6 Medical Mission Institute, 97074 Würzburg, Germany; simone_kann@hotmail.com

7 Infectious Disease Epidemiology Department, Bernhard Nocht Institute for Tropical Medicine Hamburg, 20359 Hamburg, Germany; dekker@bnitm.de (D.D.); may@bnitm.de (J.M.)

8 Department of Hospital Hygiene \& Infectious Diseases, University Medicine Göttingen, 37075 Göttingen, Germany

* Correspondence: ulrike.loderstaedt1@med.uni-goettingen.de

+ Hagen Frickmann and Ulrike Loderstädt contributed equally to this work.

\begin{abstract}
This study aimed to assess standard and harsher nucleic acid extraction schemes for diagnostic helminth real-time PCR approaches from stool samples. A standard procedure for nucleic acid extraction from stool and a procedure including bead-beating as well as proteinase $\mathrm{K}$ digestion were compared with group-, genus-, and species-specific real-time PCR assays targeting helminths and nonhelminth pathogens in human stool samples. From 25 different in-house and commercial helminth real-time PCR assays applied to 77 stool samples comprising 67 historic samples and 10 external quality assessment scheme samples positively tested for helminths, higher numbers of positive test results were observed after bead-beating-based nucleic acid extraction for 5/25 (20\%) real-time PCR assays irrespective of specificity issues. Lower cycle threshold values were observed for one real-time PCR assay after the standard extraction scheme, and for four assays after the beadbeating-based scheme. Agreement between real-time PCR results after both nucleic acid extraction strategies according to Cohen's kappa ranged from poor to almost perfect for the different assays. Varying agreement was observed in eight nonhelminth real-time PCR assays applied to 67 historic stool samples. The study indicates highly variable effects of harsh nucleic acid extraction approaches depending on the real-time PCR assay used.
\end{abstract}

Keywords: helminths; stool samples; consensus; nematodes; trematodes; cestodes; qPCR; test comparison

\section{Introduction}

Although the global burden of helminth infections is still considerable [1-11], reliable microscopic diagnosis requires experience that is scarcely available in peripheral laboratories in settings of nonendemicity apart from reference centers. An investigator-independent, standardized screening option for both individual diagnosis and surveillance purposes is therefore desirable. 
Multiple genus- and species-specific PCRs and real-time PCRs [12-28] have been introduced for helminths. A cox-gene-based group-specific real-time PCR approach targeting nematodes and cestodes has been described [29], as has a 28S-rRNA-gene-based approach targeting the trematode group [30].

While superior sensitivity of real-time PCR for protozoan parasites compared to microscopy is considered to be well established [31], reliability of diagnostic real-time PCR for helminths is believed to depend on the harshness of nucleic acid extraction from strong-shelled eggs or cuticle cells [32-34]. However, assessments of nucleic acid extraction schemes are usually performed only for specific helminth species, so it is difficult to draw general conclusions.

In the present study, a standard nucleic acid extraction scheme was compared with a harsh, bead-beating-based extraction protocol and applying multiple different helminth real-time PCRs. This was done in order to assess whether and to what extent increased sensitivity after harsh extractions is a general or a species-dependent effect.

\section{Materials and Methods}

\subsection{Study Design and Sample Materials \\ 2.1.1. Study Design}

The study was performed as a head-to-head comparison of two different nucleic acid extraction procedures regarding their effects on various diagnostic helminth real-time PCRs. Thereby, it was organized in a stepwise algorithm. In a first step, the test characteristics of the applied in-house helminth real-time PCRs were investigated. In a second step, the primary objective of the study, i.e., the comparative effects of nucleic acid extraction by either the QiaAMP DNA stool mini kit (Qiagen, Hilden, Germany) or a harsher, bead beating-based nucleic extraction procedure, was assessed. In a third step, the effects of the harsh bead beating-based nucleic extraction procedure on real-time PCRs targeting nonhelminth pathogens like bacteria and protozoa, which are also of diagnostic interest for the assessment of human stool samples in the medical diagnostic laboratory, were analyzed in comparison to the QiaAMP DNA stool mini kit-based nucleic acid extraction scheme. This was done to exclude harmful effects of such a harsh approach on the quality of DNA of less robust nonhelminth pathogens. The different steps are visualized in Diagram 1 and described in the following.

Diagram 1: Flowchart of the stepwise experimental approach.

1. Estimating the test characteristics of the applied in-house real-time PCRs:

- $\quad$ Specificity testing of the group-specific helminth real-time PCRs with helminth DNA

- $\quad$ Sensitivity and specificity testing of the group specific helminth real-time PCRs with DNA from stool samples positive in genus- and species-specific helminth real-time PCR after DNA extraction applying the QiaAMP DNA Stool Mini Kit

- Sensitivity and specificity testing of the in-house helminth real-time PCRs with DNA from stool samples positive for helminth eggs or larvae in microscopy after DNA extraction applying the QiaAMP DNA Stool Mini Kit

2. Comparing the effects of nucleic acid extraction applying the QiaAMP DNA Stool Mini Kit and a harsher bead beating-based nucleic extraction scheme with:

- Historic residual stool samples which had been positive before in any helminth real-time PCR after nucleic acid extraction using the QiaAMP DNA Stool Mini Kit

- Stool samples from a Dutch external quality assessment scheme for helminths

3. Comparative assessment of the effects of nucleic acid extraction applying the QiaAMP DNA Stool Mini Kit and a harsher bead beating-based nucleic extraction scheme on nonhelminth pathogens which might also be relevant in the medical routine diagnostic laboratory with:

- Historic residual stool samples which had been positive before in any bacteria real-time PCR after nucleic acid extraction using the QiaAMP DNA Stool Mini Kit

- Historic residual stool samples which had been positive before in any protozoa real-time PCR after nucleic acid extraction using the QiaAMP DNA Stool Mini Kit 


\subsubsection{Specificity Testing of the Group-Specific Helminth Real-Time PCRs with Helminth DNA}

As a first step, all group specific helminth real-time PCRs as described below were assessed with helminth DNA of Trichinella spiralis, Fasciola hepatica, Toxocara canis, Toxocara cati, Angyostrongylus vasorum, Dipylidium caninum, Schistosoma mansoni, and Strongyloides ratti, respectively, to exclude or confirm specificity limitations. Helminth tissue was provided from specimen collections from the institutions of the coauthors and the colleagues listed in the acknowledgements. Nucleic acid extraction was performed with the harsh bead beating-based procedure as described below.

2.1.3. Sensitivity and Specificity Testing of the Group Specific Helminth Real-Time PCRs with DNA from Stool Samples Positive in Genus- and Species-Specific Helminth Real-Time PCR after DNA Extraction Applying the QiaAMP DNA Stool Mini Kit

As a second step, both sensitivity and specificity of the group specific helminth real-time PCRs were analyzed using 96 residual DNA volumes from stool samples of patients with real-time PCR-confirmed enteric helminth infections. As negative controls, 100 residual nucleic acid extractions from stool samples of German citizens without history of travel and thus a very low likelihood of being infected with helminths were applied. Nucleic acids had been extracted using the QiaAMP DNA stool Mini Kit (Qiagen, Hilden, Germany). The eluates had been stored at $-80{ }^{\circ} \mathrm{C}$ prior to the assessment for time periods between few months and more than 10 years.

2.1.4. Sensitivity and Specificity Testing of the in-House Helminth Real-Time PCRs with DNA from Stool Samples Positive for Helminth Eggs or Larvae in Microscopy after DNA Extraction Applying the QiaAMP DNA Stool Mini Kit

Prior to the nucleic acid extraction assessment comparisons, the applied in-house stool real-time PCRs for helminths as detailed below were tested with a small number of 37 nucleic acid extractions from microscopically positive stool samples from returning travelers with gastrointestinal symptoms after tropical journeys assessed at the Bernhard Nocht Institute for Tropical Medicine Hamburg, Germany. The samples had been collected within an eight-year interval (2006-2014) and extracted using the QiaAMP DNA stool mini kit (Qiagen). The eluates had then been stored at $-80{ }^{\circ} \mathrm{C}$. Native stool was no longer available from those samples, but the eluates were assessed in order to obtain an impression of baseline sensitivity of the applied real-time PCRs. Parasite microscopy that had been performed on the fresh stool samples was based on standard approaches including enrichment using sodium acetate-acetic acid-formalin solution (SAF) [35-37] and extended by Baermann's method [38,39] in case of suspicion of Strongyloides infection. In fact, Strongyloides larvae had been seen after enrichment according to the Baermann method only. In detail, the 37 microscopically positive stool samples, i.e., samples in which at least one helminth egg or one helminth larva with potential pathogenic relevance for human patients had been microscopically observed, included had shown 29 nematode stages comprising 13 samples with Strongyloides stercoralis larvae; five with hookworm eggs; four with Ascaris lumbricoides eggs; three with Trichuris trichiura eggs; one with A. lumbricoides and T. trichiura eggs; as well as three with Enterobius vermicularis eggs; five samples with trematode eggs comprising one with small trematode eggs (suggestive of Clonorchis sinensis/Opisthorchis viverrini) and four with Schistosoma mansoni eggs; as well as three with Hymenolepis nana eggs as representatives of cestodes. Microscopical quantification had not been performed, i.e., the exact number of observed helminth eggs or helminth larvae per assessed microscopical slide had not been recorded. In the investigators' experience, however, helminth loads in travel returnees are usually low.

2.1.5. Comparing the Effects of Nucleic Acid Extraction Applying the QiaAMP DNA Stool Mini Kit and a Harsher Bead Beating-Based Nucleic Extraction Scheme

After this, the study was conducted as a head-to-head comparison of nucleic acid extractions using 67 residual materials from stool samples that had shown positive real-time PCR signals for helminths in previous diagnostic assessments at the Bundeswehr Hospital 
Hamburg, Department for Microbiology and Hygiene, external site at the Bernhard Nocht Institute for Tropical Medicine Hamburg, Germany. Those stool samples had originally been obtained for diagnostic screening purposes from soldiers and policemen returning from tropical deployments, migrants travelling under poor hygiene conditions, and study participants from resource-poor tropical settings over the course of 13 years (2006-2019). No microscopic results were available for those assessed stool samples. Both bead beatingfree and bead beating-based nucleic acid extractions as detailed below and residual stool samples had been stored at $-80{ }^{\circ} \mathrm{C}$ prior to further assessment. Ethical clearance allowed only the anonymous use of residual sample materials for test comparison purposes in a fully anonymized way. Accordingly, no detailed patient-related information can be presented. In addition to the 67 diagnostic samples used, 10 stool samples from a Dutch external quality assessment scheme for helminths as described elsewhere [40] were included in the comparison. From the residual sample collection in Hamburg, a further 67 residual materials from stool samples that had shown positive real-time PCR signals in nonhelminth real-time PCRs were chosen to assess the effects of both nucleic acid extraction schemes on real-time PCRs targeting bacterial and protozoan pathogens.

2.1.6. Effects of Bead-Beating-Based Nucleic Acid Extraction on Nonhelminth Real-Time PCR Targets

To assess the effects of harsh bead-beating-based nucleic acid extraction on nonhelminth real-time PCR targets, the 67 residual stool samples with positive in-house real-time multiplex real-time PCR results for the enteroinvasive bacteria Salmonella spp., Shigella spp./enteroinvasive Escherichia coli (EIEC), Campylobacter jejuni, or Yersinia spp. [41] as well as the enteropathogenic protozoa Entamoeba histolytica, Giardia duodenalis, Cryptosporidium spp., or Cyclospora spp. [28] were subjected to bead-beating-based nucleic acid extraction with subsequent repetition of the real-time PCRs. Similar as described above for the helminth assessments, the applied residual DNA samples had been stored frozen at $-80{ }^{\circ} \mathrm{C}$ over the course of 13 years (2006-2019) as well.

\subsection{Nucleic Acid Extraction}

For the initial diagnostic assessment, nucleic acid from all samples had been extracted using the QiaAMP DNA Stool Mini Kit (Qiagen, Hilden, Germany) as described by the manufacturer without particular focus on specialized procedures for parasite DNA (later referred to as "without bead beating"). If any helminth real-time PCR signal had been detectable in the initial diagnostic process and residual stool material was still available stored at $-80^{\circ} \mathrm{C}$, nucleic acid from these residual materials were extracted using polyvinylpyrrolidone pretreatment, bead-beating using garnet beads $(0.7-1.2 \mathrm{~mm}$, Biolabproducts, Bebensee, Germany) with a TissueLyser LT device (Qiagen) for $3 \mathrm{~min}$ at $30 \mathrm{~s}^{-1}$, proteinase K digestion, and QiaAMP spin column extraction (Qiagen) as described previously [33]. This procedure is later referred to as "with bead beating". Identical elution volumes as in the QiaAMP DNA Stool Mini Kit-based approach were achieved. To ensure sufficient sample volumes for the real-time PCR assays, photometric assessment of DNA concentrations within the samples using a Pico 100 Picodrop Microliter Spectrophotometer (Picodrop Ltd., Hinxton, UK) according to the manufacturer's instructions was only performed from remaining residual DNA samples after the real-time PCRs had been completed.

\subsection{Helminth Real-Time PCR Protocols}

The total of 25 genus- and species-specific helminth real-time PCRs used comprised in-house real-time PCRs targeting Ancylostoma spp., A. lumbricoides, E. vermicularis, $H$. nana, Necator americanus, Schistosoma spp., S. stercoralis, Taenia saginata, T. solium, and T. trichiura as well as commercial real-time PCRs (Tib MolBiol, Berlin, Germany, product codes of the kits: MDx_64-0710-96, MDx_50-0705-96, MDx_50-0714-96, MDx_58-070796, MDx_64-0709-96, MDx_61-0708-96, MDx_58-0639-96) targeting Ancylostoma spp., A. lumbricoides, E. vermicularis, H. nana, N. americanus, Schistosoma spp., and S. stercoralis, as recently described [28]. Additionally, specific multi-copy-target real-time PCRs for $S$. 
mansoni complex [42] and S. haematobium complex [43], which were originally designed for application with blood samples, were used as detailed previously [42,43] with DNA from stool. Group-specific helminth real-time PCRs targeting nematodes, trematodes, and cestodes were applied as described $[29,30]$ as simplex assays. In addition, groupspecific assays for nematodes, trematodes, and cestodes targeting the 18S-rRNA-gene were run (details on the group-specific assays are provided in the Tables 1 and 2). Thereby, genus- and species-specific real-time PCRs were run on RotorGene Q cyclers (Qiagen, Hilden, Germany), group-specific real-time PCRs on RotorGene 6000 cyclers (Qiagen). Real-time PCRs from eluates after different nucleic acid extraction procedures were not continuously assessed in parallel in the same runs but were distributed on the runs without a specific order.

\subsection{Inclusion and Exclusion Criteria}

Samples were included if at least one positive pathogen-specific real-time PCR result had been observed during routine diagnostic assessment. There were no exclusion criteria, so samples were also included if residual sample material was insufficient for all assessments.

\subsection{Statistical Assessment}

Due to the low sample count of this proof-of-principle assessment, statistical approaches were basically descriptive. In detail, Cohen's kappa was calculated to assess the agreement between the extraction schemes for each individual real-time PCR target. The interpretation standards as described [44] with the categories poor (below 0.00), slight $(0.00-0.20)$, fair $(0.21-0.40)$, moderate $(0.41-0.60)$, substantial (0.61-0.80), and almost perfect (0.81-1.00) were applied. Significance for differences regarding the qualitative results, i.e., positive or negative real-time PCR results, was calculated by applying tests for proportions. Normal distribution of the $\mathrm{Ct}$ (cycle threshold) values was assumed. This assumption was proven applying Shapiro-Wilk testing with Bonferroni correction for multiple testing. There was no evidence for rejecting the assumption of a normal distribution of the $\mathrm{Ct}$ values. For the descriptive comparison of $\mathrm{Ct}$ values, the unpaired T-test was applied without correction for multiple testing. Welch's adjustment for unequal variances between the groups was applied if the hypothesis of equal variances was rejected (significance level 0.05). Unpaired T-testing was chosen as a consequence of different processing of the assessed patient materials. In detail, pretreatment of the patient materials, i.e., the nucleic acid extraction procedure, is a prerequisite to obtain analyzable samples. In turn, the sample itself is not assessed first with one method and then again with the other method, but rather two samples are generated from one patient material by different pretreatment approaches. Only then, they represent analyzable samples by themselves. Accordingly, they were considered as two different samples, favoring the use of unpaired testing. The calculations were performed using the software Stata/IC 15.1 for Mac 64-bit Intel (College Station, TX, USA).

\subsection{Ethical Clearance}

Ethical clearance, provided for blinded use of residual materials for test comparison and evaluation purposes, was granted on 11 March 2019 by the ethics committee of the Medical Association of Hamburg, Germany (registration number WF-011/19) in line with national laws without requirement for informed consent. The authors assert that all procedures contributing to this work comply with the Helsinki Declaration of 1975, as revised in 2008. 
Table 1. First real-time PCR platform with consensus real-time PCRs for nematodes, trematodes, and cestodes.

\begin{tabular}{|c|c|c|c|}
\hline Oligonucleotide & Oligonucleotide Name & SEQUENCE & Reference \\
\hline \multicolumn{4}{|c|}{ Nematode consensus real-time PCR 1} \\
\hline Forward Primer & pan_nematode_cox1_692F & 5'-TGT-CTT-TAC-CWG-TTT-TRG-CTG-G-3' & \multirow{3}{*}{ [29] } \\
\hline Reverse Primer & pan_nematode_cox1_835R & 5'-CCG-AAA-GCA-GGY-AAA-ATH-ARA-A-3' & \\
\hline Probe & pan_nematode_cox1_795P & 5'-FAM-TCA-RCA-TTT-RTT-TTG-RTT-TTT-TGG-TCA-TCC-BHQ1-3' & \\
\hline \multicolumn{4}{|c|}{ Trematode consensus real-time PCR 1} \\
\hline Forward Primer & pan_trematode_28S_2F & 5'-AGG-CAA-TGT-GGT-GTT-YAG-GT-3' & \multirow{3}{*}{ [30] } \\
\hline Reverse Primer & pan_trematode_28S_168R & 5'-CAC-AAA-CAA-CCC-GAC-TCC-AA-3' & \\
\hline Probe & pan_trematode_28S_T & 5'-FAM-TGG-CCC-AND-GAG-GGT-GAA-AGG-C-BHQ1-3' & \\
\hline \multicolumn{4}{|c|}{ Cestode consensus real-time PCR 1} \\
\hline Forward Primer & pan_cestode_cox1_82F & 5'-TGG-GTT-ATT-GTT-TGC-TAT-GTT-TTC-WA-3' & \multirow{3}{*}{ [29] } \\
\hline Reverse Primer & pan_cestode_cox1_209R & 5'-CCC-CTA-TTA-TCA-TAG-TAA-CMG-AAC-TAA-A-3' & \\
\hline Probe & pan_cestode_cox1_143P & 5'-FAM-ATG-TTT-ACG-GTT-GGG-TTR-GAT-GTK-AAG-BHQ1-3' & \\
\hline & \multirow{8}{*}{\multicolumn{3}{|c|}{$\begin{array}{l}\text { Eco-R1-restriction-site-TTATTGGTTTTGTCTTTACCTGTTTTAGCTGGTGCTATTACTATGTTGTTAATTGATCGTAA } \\
\text { TTTTAATGGTTCTTTTTTTGATCCTAGTTTTGGTGGTAATCCTTTGATTTATCAGCATTTGTTTTGGTTTTTTGGTC } \\
\text { ATCCAGAAGTTTATATTTTAATTTTACCTGCTTTCGGTATTATTAGT-Eco-R1-restriction side-TTGGCTTTTATGGG } \\
\text { TTATTGTTTGCTATGTTTCAATAGTATGTTAGGAAGAAGTGTGTGAGGACATCATATGTTTACGGTTGGGTT } \\
\text { AGATGTTAAGACGGCTGTATTTTTAGTTCTGTT ACTATGATAATTGGAGTGCCTACGGG-Eco-R1-restriction-site- } \\
\text { ATTGGTCACTAGGCAATGTGGTGTTCAGGTCGTTCCGCGGAGGTGCTGCTCCATTCCAAGTCCAGCAATGAG } \\
\text { TACGGTAATGCTGACATGGCCCAAAGAGGGTGAAAGGCCCGTTGGGGTGGAGAGGCAGAAATGACAGCACC } \\
\text { TTCCTGGATAGACCTTGGAGTCGGGT TGTTTGTGAATGCAGCCCAA-Eco-R1-restriction-site. }\end{array}$}} \\
\hline & & & \\
\hline & & & \\
\hline positive-control & & & \\
\hline plasmid sequence & & & \\
\hline insert & & & \\
\hline & & & \\
\hline & & & \\
\hline
\end{tabular}

The real-time PCRs were run in $20 \mu \mathrm{L}$ reaction volumes containing $10 \mu \mathrm{L}$ HotStar master mix (Qiagen, Hilden, Germany), $5.0 \mathrm{mM}$ total $\mathrm{MgCl}_{2}, 5 \times 10^{-7} \mathrm{~mol}$ of each primer, $2 \times 10^{-7} \mathrm{~mol}$ probe, and $4.0 \mu \mathrm{L}$ DNA eluate on a RotorGene 6000 cycler (Qiagen). The steps were activation at $95^{\circ} \mathrm{C}$ for $15 \mathrm{~min}$ (minutes) followed by 55 cycles of $45 \mathrm{~s}$ (seconds) denaturation at $95^{\circ} \mathrm{C}$ and $45 \mathrm{~s}$ annealing and elongation at $55^{\circ} \mathrm{C}$. After this, the tubes were cooled to $40^{\circ} \mathrm{C}$ for an additional $20 \mathrm{~s}$ before the run was finished.

Table 2. Second real-time PCR platform with consensus real-time PCRs for nematodes, trematodes, and cestodes.

\begin{tabular}{|c|c|c|c|c|}
\hline Oligonucleotide & Oligonucleotide Name & Sequence & In-Silico Coverage & Genbank Accession Numbers \\
\hline \multicolumn{5}{|c|}{ Nematode consensus real-time PCR 2} \\
\hline Forward Primer & Nem1Gr1-6-7-Go S & $\begin{array}{l}\text { 5'-GAA-TYC-CTA-GTA-ART- } \\
\text { GTG-AGT-CAT-C-3' }\end{array}$ & $\begin{array}{l}\text { As detailed for the sum of the } \\
\text { reverse primers }\end{array}$ & n.a. \\
\hline \multirow{5}{*}{ Reverse Primer } & Nem1Gr2 A & $\begin{array}{l}\text { 5'-GCC-TCT-SGA-TAT-TGC- } \\
\text { TCA-GT-3' }\end{array}$ & $\begin{array}{l}\text { Strongyloides stercoralis, Strongyloides } \\
\text { fuelleborni, Parastrongyloides trichosuri }\end{array}$ & $\begin{array}{c}\text { AB923885.1, LM523351.1, } \\
\text { AB453320.1, AB453322.1, AB821045.1 }\end{array}$ \\
\hline & Nem_Ancylo B & $\begin{array}{c}5^{\prime} \text {-CTC-GAT-ATA-GCA-GGC- } \\
\text { CGA-3 }{ }^{\prime}\end{array}$ & $\begin{array}{l}\text { Ancylostoma caninum, Ancylostoma } \\
\text { duodenale, Ancylostoma ceylanicum }\end{array}$ & $\begin{array}{c}\text { AJ920347.2, MH508247.1, } \\
\text { EU344798.1, DQ464371, MH508245.1, } \\
\text { LC036567.1 }\end{array}$ \\
\hline & Nem1X B & $\begin{array}{l}5^{\prime} \text {-GCC-TCG-AAA-CAG- } \\
\text { CAG-TCY-SC-3' }\end{array}$ & $\begin{array}{c}\text { Enterobius vermicularis, Dracunculus } \\
\text { medinensis, Dracunculus lutrae, } \\
\text { Dracunculus insignis, Brugia malayi, } \\
\text { Dirofilaria repens, Loa, Anisakis simplex, } \\
\text { Ascaris lumbricoides, Baylisascaris } \\
\text { schroederi, Baylisascaris ailuri, Baylisascaris } \\
\text { transfuga, Baylisascaris procyonis, } \\
\text { Contracaecum multipapillatum, Toxacara } \\
\text { cati, Toxocara canis, Ancylostoma caninum, } \\
\text { Ancylostoma duodenale, Ancylostoma } \\
\text { ceylanicum, Angiostrongylus cantonensis, } \\
\text { Angiostrongylus chabaudi, Angiostrongylus } \\
\text { costaricensis, Angiostrongylus vasorum, } \\
\text { Necator americanus, Oesophagostomum } \\
\text { aculeatum, Trichostrongylus colubriformis, } \\
\text { Gongylonema pulchrum }\end{array}$ & $\begin{array}{c}\text { AB626660, HQ646164, JF934731.1, } \\
\text { KF770013.1, KF770015.1, JF934737.1, } \\
\text { AY947719.1, AF100621, } \\
\text { XM_001893642.1, AF036588.1, } \\
\text { AB973229, MG657262.1, } \\
\text { MH981971.1, AB973229, } \\
\text { XR_002251421, MF072711.1, } \\
\text { LN600407.1, U94366.1, JN256992.1, } \\
\text { JN256991.1, U94369.1, KU050692.1, } \\
\text { MG696302.1, EF180059.1, JN256973.1, } \\
\text { AF036608.1, U94382.1, AJ920347.2, } \\
\text { MH508247.1, EU344798.1, DQ464371, } \\
\text { MH508245.1, LC036567.1, } \\
\text { AY295804.1, KX378963.1, } \\
\text { KX378964.1, AJ920365.1, AJ920348.1, } \\
\text { AY295811.1, AB677956.1, AJ920350.1, } \\
\text { AB646055.1, LC388753.1, AB495401.2 }\end{array}$ \\
\hline & Nem1Gr9 B & $\begin{array}{c}\text { 5'-CGG-CAT-CGG-TCC- } \\
\text { AAA-3' }\end{array}$ & Trichuris trichiura & $\begin{array}{l}\text { GQ352553.1, GQ352553.1, } \\
\text { GQ352554.1 }\end{array}$ \\
\hline & Nem1Gr10 B & $\begin{array}{c}5^{\prime}-\mathrm{CTA}-\mathrm{CTG}-\mathrm{GCG}-\mathrm{CYA}-\mathrm{GTC}- \\
\text { AAA-A-3' }\end{array}$ & $\begin{array}{l}\text { Trichinella britovi, Trichinella nativa, } \\
\text { Trichinella nelsoni, Trichinella pupae, } \\
\text { Trichinella spiralis }\end{array}$ & $\begin{array}{c}\text { AY851257.1, KP307966, AY851261.1, } \\
\text { AY851263.1, KU725991.1 }\end{array}$ \\
\hline Probe & Nem1 TM & $\begin{array}{c}\text { 5'-FAM-TAC-KTC-CCT-GCC- } \\
\text { MTT-TGT-ACA-CAC-C- } \\
\text { BHQ1-3' }\end{array}$ & $\begin{array}{l}\text { As detailed for the sum of the reverse } \\
\text { primers }\end{array}$ & n.a. \\
\hline
\end{tabular}


Table 2. Cont.

\begin{tabular}{|c|c|c|c|c|}
\hline Oligonucleotide & Oligonucleotide Name & Sequence & In-Silico Coverage & Genbank Accession Numbers \\
\hline \multicolumn{5}{|c|}{ Trematode consensus real-time PCR 2} \\
\hline Forward Primer & Trem1 F & $\begin{array}{l}\text { 5'-WGA-GGC-TCC-GTA- } \\
\text { ATT-CGA-3' }\end{array}$ & $\begin{array}{l}\text { As detailed for the sum of the reverse } \\
\text { primers }\end{array}$ & n.a. \\
\hline \multirow{2}{*}{ Reverse Primer } & Trem1 Gr1 R & $\begin{array}{l}5^{\prime} \text {-TGC-GAY-CGC-ACK- } \\
\text { ACC-C-3' }\end{array}$ & $\begin{array}{l}\text { Schistosoma intercalatum, Schistosoma } \\
\text { japonicum, Schistosoma mansoni, } \\
\text { Schistosoma mekongi }\end{array}$ & $\begin{array}{l}\text { DQ354363.1, U42564.1, AY157226.1, } \\
\text { JF721335.1, U65657.1, X53047.1, } \\
\text { XR_001974584.1, AY157228.1 }\end{array}$ \\
\hline & Trem1Gr3-6 R & $\begin{array}{c}5^{\prime}-\mathrm{CRY}-\mathrm{AGC}-\mathrm{CAT}-\mathrm{SCG}-\mathrm{ACC}- \\
\text { C-3' }\end{array}$ & $\begin{array}{c}\text { Fasciola gigantica, Gastrodiscoides hominis, } \\
\text { Paragonimus westermani, Nanophyetus } \\
\text { salmincola, Paragonimus vietnamesi, } \\
\text { Paragonimus kellicotti }\end{array}$ & $\begin{array}{l}\text { AJ004804.1, MF077354.1, AJ011942.1, } \\
\text { JX678223.1, KF781291.1, AY628372.1, } \\
\text { AJ287556.1, AY222140.1, AY222138.1, } \\
\text { KX990282.1, LT855189.1, HQ900670.1 }\end{array}$ \\
\hline \multirow[t]{2}{*}{ Probe } & Trem1 TM & $\begin{array}{l}\text { 5'-FAM-YCA-ACT-ACG- } \\
\text { AGC-TTT-TKA-ACT-GCA- } \\
\text { RCA-ACT-BHQ1-3' }\end{array}$ & $\begin{array}{l}\text { As detailed for the sum of the reverse } \\
\text { primers }\end{array}$ & n.a. \\
\hline & \multicolumn{4}{|c|}{ Cestode consensus real-time PCR 2} \\
\hline \multirow[t]{3}{*}{ Forward Primer } & Ces1Gr2-4 F & $\begin{array}{l}\text { 5'-GGT-TTA-TTG-GAT-CRT- } \\
\text { RCC-CGT-TAA-A-3' }\end{array}$ & $\begin{array}{c}\text { Dipylidium caninum, Diphyllobothrium } \\
\text { latum, Diphyllobothrium ditrenum, } \\
\text { Diphyllobothrium balaenopterae, } \\
\text { Diphyllobothrium cameroni, } \\
\text { Diphyllobothrium pacificum, Diplogonoporus } \\
\text { grandis, Raillietina spp., Raillietina australis, } \\
\text { Raillietina chiltoni, Raillietina sonini }\end{array}$ & $\begin{array}{c}\text { AB731643.1, MG582184.2, } \\
\text { MG582181.1, MG582183.1, } \\
\text { KF218246.1, KY552781.1, KF218250.1, } \\
\text { KY552787.1, KY552792.1, } \\
\text { KY552796.1, DQ925310.2, } \\
\text { KF218253.1, KY945917.1, } \\
\text { HG315734.1, AB353272.1, } \\
\text { EU665464.1, EU665466.1, } \\
\text { EU665467.1, AF286980.1, } \\
\text { AY382313.1, EU665468.1 }\end{array}$ \\
\hline & Ces1Gr5 F & $\begin{array}{l}\text { 5'-TGG-TTT-ATT-GGA-TCR- } \\
\text { TAC-TCG-TTA-AA-3' }\end{array}$ & Hymenolepis diminuta, Hymenolepis nana & $\begin{array}{c}\text { AF124475.1, AF286983.1, JX310720.1, } \\
\text { AF461124.1, AY193873.1, AY193874.1, } \\
\text { AY193875.1 }\end{array}$ \\
\hline & Ces1Gr6-6a F & $\begin{array}{l}\text { 5'-GTT-TAT-TGG-ATC-GTA- } \\
\text { CCC-GTT-AAR-3' }\end{array}$ & $\begin{array}{c}\text { Raillietina echinobothrida, Taenia saginata, } \\
\text { Taenia solium, Taenia multiceps, Bertiella } \\
\text { studeri }\end{array}$ & $\begin{array}{c}\text { MH119095.1, AB731616.1, } \\
\text { JQ609338.1, DQ768166.1, U88076.1, } \\
\text { AB731615.1, DQ157224.1, } \\
\text { AB731621.1, GQ260089.1, } \\
\text { GU323707.1 }\end{array}$ \\
\hline Reverse Primer & Ces1 A & $\begin{array}{c}\text { 5'-GGT-TGG-CTT-CTG-DTC- } \\
\text { TAA-TAA-GTG-3' }\end{array}$ & $\begin{array}{l}\text { As detailed for the sum of the forward } \\
\text { primers }\end{array}$ & n.a. \\
\hline Probe & Ces1 TM & $\begin{array}{l}\text { 5'-FAM-AGA-GCT-AAT- } \\
\text { ACA-TGC-CHY-GAW-GCC- } \\
\text { CTG-AC-BHQ1-3' }\end{array}$ & $\begin{array}{l}\text { As detailed for the sum of the forward } \\
\text { primers }\end{array}$ & n.a. \\
\hline $\begin{array}{l}\text { Combined } \\
\text { positive-control } \\
\text { plasmid } \\
\text { sequence insert }\end{array}$ & \multicolumn{4}{|c|}{$\begin{array}{l}\text { Eco-R1-restriction-site-ATTGAAAACATTACGTAACTGGGAGTGAAAATTGCAATTATTTTTCATGAACGAGGAATTCCAAGTAAACGT } \\
\text { AAGTCATTAGCTTACATTGATTACGTCCCTGCCCTTTGTACACACCGCCCGTCGCTGCCCGGAACTGAGCAATATCCAGAGGCAGG } \\
\text { AAGAGATGTAATAA- Eco-R1-restriction-site-AATACGGATACGGGACTCACTAGAGGCTCCGTAATCGAATGAGTACAATTTAAATCC } \\
\text { TTTAACGAGGATCAACTGGAGGGCAAGTCTGGTGCCAGCAGCCGCGGTAACTCCAGCTCCAGAAGCGTATATTAAAGTTGTTGC } \\
\text { AGTTCAAAAGCTCGTAGTTGGATCTGGGTCGCATGGCTACATGCCGTCGCTCGTGGGTCTGGCCTGGTTAC-Eco-R1-restriction-site- } \\
\text { CTATGGTTTATTGGATCGTACCCGTTAAATGGGTAACTGTAATAACTCTAGAGCTAATACATGCCCCGATGCCCTGACTCTGTTAGCC } \\
\text { TGCTGCTGCTTGCTTGTGTGTGGGTGGTGGCGGGTAGGCAGGGTGTGGGTGCACTTATTAGATCAGAAGCCAACCAACTGCTCG } \\
\text { AACGTTGCGTGTGCAGTCCACTGCTGTAGGCGTGCGTGCGGGTGTTGAGAGGAGACCGCTTCTGGTGACTCTGGATAATTGTTA } \\
\text { CAGATCGCAGTCGGCCTTGAGTCGGCGACGGGTCCTTCAA-Eco-R1-restriction-site }\end{array}$} \\
\hline
\end{tabular}

The real-time PCRs were run in $20 \mu \mathrm{L}$ reaction volumes containing $10 \mu \mathrm{L}$ HotStar master mix (Qiagen, Hilden, Germany), $5.0 \mathrm{mM}$ total $\mathrm{MgCl}_{2}, 2.5 \times 10^{-7} \mathrm{~mol}$ of each primer, $6 \times 10^{-6} \mathrm{~mol}$ probe, and $2.0 \mu \mathrm{L}$ DNA eluate on a RotorGene 6000 cycler (Qiagen, Hilden, Germany). The steps were activation at $95{ }^{\circ} \mathrm{C}$ for $15 \mathrm{~min}$ followed by 45 cycles of $15 \mathrm{~s}$ denaturation at $95^{\circ} \mathrm{C}, 30 \mathrm{~s}$ annealing at $60^{\circ} \mathrm{C}$ and $30 \mathrm{~s}$ elongation at $72{ }^{\circ} \mathrm{C}$. After this, the tubes were cooled to $40^{\circ} \mathrm{C}$ for an additional $20 \mathrm{~s}$ before the run was finished. Note that n.a. $=$ not applicable.

\section{Results}

\subsection{Results of the Group Specific Helminth Real-Time PCRs with Helminth DNA}

When applied with helminth DNA, individual cross-reactions of the group specific helminth real-time PCRs were seen. Such cross-reactions were associated with comparably high cycle threshold $(\mathrm{Ct})$ values. Details of the $\mathrm{Ct}$ values of observed reactions and crossreactions with nucleic acid extractions of helminths are shown in Table 3.

3.2. Sensitivity and Specificity Testing of the Group-Specific Helminth Real-Time PCRs with DNA Residuals from Stool Samples without Available Microscopic Results

Applied with 96 residual DNA samples extracted from stool of patients with previous real-time PCR-based diagnosis of enteric helminth infections, imperfect sensitivity and specificity of the group specific helminth real-time PCRs was calculated. In detail, application of the group-specific real-time PCRs with samples positive in genus- or species specific helminth real-time PCRs (in-house protocols according to [28]) after QiaAMP DNA Stool 
Mini Kit-based extraction without microscopic control gave the results as detailed in the Table A1 of Appendix A and summarized in Table 4. Of note, the group specific real-time PCRs for nematodes, trematodes, and cestodes did not show any cross-reactions with stool samples of 100 German individuals without any history of travel, making intestinal carriage of helminths highly unlikely, suggesting acceptable overall specificity of those assays.

Table 3. Ct values of reactions and cross-reactions of group specific helminth real-time PCRs with nucleic acid extractions of helminths.

\begin{tabular}{|c|c|c|c|c|c|c|c|}
\hline $\begin{array}{l}\text { Sample } \\
\text { Number }\end{array}$ & Species & $\begin{array}{l}\text { Ct Values of } \\
\text { Nematode } \\
\text { Consensus } \\
\text { Real-Time } \\
\text { PCR } 2\end{array}$ & $\begin{array}{l}\text { Ct Values of } \\
\text { Trematode } \\
\text { Consensus } \\
\text { Real-Time } \\
\text { PCR } 2\end{array}$ & $\begin{array}{l}\text { Ct Values of } \\
\text { Cestode } \\
\text { Consensus } \\
\text { Real-Time } \\
\text { PCR } 2\end{array}$ & $\begin{array}{l}\text { Ct Values of } \\
\text { Nematode } \\
\text { Consensus } \\
\text { Real-Time } \\
\text { PCR } 1\end{array}$ & $\begin{array}{l}\text { Ct Value of } \\
\text { Trematode } \\
\text { Consensus } \\
\text { Real-Time } \\
\text { PCR } 1\end{array}$ & $\begin{array}{l}\text { Ct Value of } \\
\text { Cestode } \\
\text { Consensus } \\
\text { Real-Time } \\
\text { PCR } 1\end{array}$ \\
\hline TiHo1 & Trichinella spiralis & 17 & & & 42 & & \\
\hline TiHo2 & Fasciola hepatica & & 17 & & $39 *$ & 14 & \\
\hline TiHo3 & Toxocara canis & 13 & & & 14 & 24 & \\
\hline TiHo4 & Toxacara cati & 19 & $34 *$ & & 21 & 26 * & \\
\hline TiHo5 & Taenia saginata & $26 *$ & 34 * & 32 & $37 *$ & $30 *$ & 26 \\
\hline TiHo7 & Angiostrongylus vasorum & 27 & $36^{*}$ & & 19 & & \\
\hline TiHo9 & Dipylidium caninum & & & 23 & & & 13 \\
\hline Nr. 15 & Schistosoma mansoni & & 12 & & & 11 & \\
\hline Nr. 16 & Strongyloides ratti & 32 & & & 14 & & \\
\hline
\end{tabular}

${ }^{*}$ Cross-reaction.

Table 4. Sensitivity and specificity of the group specific helminth real-time PCRs as calculated from the results of 96 residual DNA samples extracted from stool of patients with previous real-time PCR-based diagnosis of enteric helminth infections (see also Appendix A, Table A1).

\begin{tabular}{ccccccc}
\hline $\begin{array}{c}\text { Sensitivity/ } \\
\text { Specificity }\end{array}$ & $\begin{array}{c}\text { Nematode } \\
\text { Consensus } \\
\text { Real-Time } \\
\text { PCR 2 }\end{array}$ & $\begin{array}{c}\text { Trematode } \\
\text { Consensus } \\
\text { Real-Time } \\
\text { PCR 2 }\end{array}$ & $\begin{array}{c}\text { Cestode } \\
\text { Consensus } \\
\text { Real-Time } \\
\text { PCR 2 }\end{array}$ & $\begin{array}{c}\text { Nematode } \\
\text { Consensus } \\
\text { Real-Time } \\
\text { PCR 1 }\end{array}$ & $\begin{array}{c}\text { Trematode } \\
\text { Consensus } \\
\text { Real-Time } \\
\text { PCR 1 }\end{array}$ & $\begin{array}{c}\text { Cestode } \\
\text { Consensus } \\
\text { Real-Time } \\
\text { PCR 1 }\end{array}$ \\
\hline $\begin{array}{c}\text { Sensitivity in } \% \\
(\mathrm{n} / \mathrm{n})\end{array}$ & $40 \%(20 / 50)$ & $84.6 \%(11 / 13)$ & $62.9 \%(22 / 35)$ & $30 \%(15 / 50)$ & $76.9 \%(10 / 13)$ & $85.7 \%(30 / 35)$ \\
\hline $\begin{array}{c}\text { Specificity in } \% \\
(\mathrm{n} / \mathrm{n})\end{array}$ & $90.6 \%(87 / 96)$ & $99.0 \%(95 / 96)$ & $97.9 \%(94 / 96)$ & $94.8 \%(91 / 96)$ & $100 \%(96 / 96)$ & $94.8 \%(91 / 96)$ \\
\hline
\end{tabular}

3.3. Sensitivity Assessment with in-House Real-Time PCRs for Stool and Standard Nucleic Acid Extractions from Microscopically Positive Stool Samples

As assessed using the nucleic acid residuals of the microscopically positive samples $(n=37)$, specificity of the in-house stool real-time PCRs for helminths after QiaAMP DNA Stool Mini Kit-extraction was generally good (96.7-100\%). In contrast, there was a broad range of sensitivity ranging from $20.7 \%$ to $100 \%$ over the different in-house real-time PCRs for stool. Details of the assessments with the microscopically positive samples including cycle threshold values are provided in Table 5. Insufficiency of sample volumes did not allow the inclusion of the Schistosoma-specific blood real-time PCRs and the commercial assays. So, this quality control assessment had to be restricted to the real-time PCR assays as shown in Table 3 and as reported previously [28]. Afterwards, the sample volumes were gone. 
Table 5. Summary of results of helminth real-time PCRs with microscopically positive samples $(n=37)$ after extraction using the QiaAMP DNA Stool Mini Kit. Sensitivity and specificity were calculated in comparison with the microscopical results of the stool samples.

\begin{tabular}{|c|c|c|c|c|c|}
\hline PCR Target & Sensitivity & Specificity & $\begin{array}{c}\text { Mean Ct } \\
\text { Value }\end{array}$ & $\begin{array}{c}\text { Standard } \\
\text { Deviation (SD) }\end{array}$ & $\begin{array}{l}\text { Median Ct } \\
\text { Value }\end{array}$ \\
\hline Pan-trematode $18 \mathrm{~S}$ rRNA gene & $60 \%(3 / 5)$ & $96.9 \%(31 / 32)^{1}$ & 25.3 & 2.6 & 25.0 \\
\hline Pan-trematode $28 \mathrm{~S}$ rRNA gene & $60 \%(3 / 5)$ & $96.9 \%(31 / 32)^{1}$ & 26.8 & 1.3 & 27.0 \\
\hline Pan-cestode 18S rRNA gene & $100 \%(3 / 3)$ & $100 \%(34 / 34)$ & 27.7 & 1.5 & 28.0 \\
\hline Pan-cestode cox gene & $100 \%(3 / 3)$ & $97.1 \%(33 / 34)^{2}$ & 32.3 & 2.6 & 31.5 \\
\hline Pan-nematode $18 S$ rRNA gene & $20.7 \%(6 / 29)$ & $100 \%(8 / 8)$ & 32.5 & 5.3 & 35.0 \\
\hline Pan-nematode cox gene & $41.4 \%(12 / 29)$ & $100 \%(8 / 8)$ & 32.6 & 3.4 & 33.5 \\
\hline Ascaris lumbricoides & $40 \%(2 / 5)$ & $100 \%(32 / 32)$ & 32.5 & 0.7 & 32.5 \\
\hline Ancylostoma spp. & $100 \%(2 / 2)$ & $100 \%(32 / 32)$ & 31.5 & 0.7 & 31.5 \\
\hline Necator americanus & $100 \%(3 / 3)$ & $100 \%(32 / 32)$ & 27.3 & 4.5 & 27.0 \\
\hline Strongyloides stercoralis & $38.5 \%(5 / 13)$ & $100 \%(24 / 24)$ & 29 & 4.4 & 28 \\
\hline Schistosoma spp. & $75.0 \%(3 / 4)$ & $96.7 \%(29 / 30)^{3}$ & 18.5 & 2.6 & 18 \\
\hline Trichurius trichiura & $33.3 \%(1 / 3)$ & $100 \%(31 / 31)$ & 27 & n.a. & 27 \\
\hline Taenia saginata & n.a. $(0 / 0)$ & $100 \%(34 / 34)$ & n.a. & n.a. & n.a. \\
\hline Taenia solium & n.a. $(0 / 0)$ & $100 \%(34 / 34)$ & n.a. & n.a. & n.a. \\
\hline Enterobius vermicularis & $50 \%(1 / 2)$ & $96.9 \%(31 / 32)^{4}$ & 27 & 1.7 & 26 \\
\hline Hymenolepis nana & $100 \%(3 / 3)$ & $100 \%(32 / 32)$ & 30 & 5.7 & 30 \\
\hline
\end{tabular}

n.a. $=$ not applicable. ${ }^{1}$ Reaction with a sample with Trichuris eggs; ${ }^{2}$ reaction with a sample with Ascaris and Trichuris eggs; ${ }^{3}$ reaction with a sample with Trichuris eggs; ${ }^{4}$ reaction with a sample with Ascaris eggs. Concordant cross-reaction of ${ }^{1}$ and ${ }^{3}$ makes the microscopical nondetection of Schistosoma eggs likely in this sample. ${ }^{5} \mathrm{SD}$ values with low absolute numbers of samples have to be interpreted with care.

\subsection{Comparison of Qualitative and Quantitative Results of Helminth Real-Time PCRs after the Two Compared Nucleic Acid Extraction Procedures}

More positive results following bead-beating-based nucleic acid extraction as suggested by significance levels $p<0.05$ were observed for in-house and commercial real-time PCR targeting A. lumbricoides and N. americanus as well as for commercial real-time PCR targeting H. nana. If Bonferroni's correction is applied [45], however, the significance is challenged. A wide range of agreement in line with the definitions applied was observed between the compared nucleic acid extraction schemes, ranging from almost perfect agreement for the cox-gene-based pan-cestode real-time PCR, both the $18 \mathrm{~S}$ and the 28S rRNA-gene based pan-trematode real-time PCRs, both the in-house and the commercial Ancylostoma spp. real-time PCRs and Schistosoma spp. real-time PCRs, and the in-house $H$. nana real-time PCR; through substantial agreement for the $18 \mathrm{~S}$ rRNA gene-based pancestode real-time PCR, both the 18S rRNA gene- and the cox-gene-based pan-trematode real-time PCRs, the in-house T. solium and S. mansoni real-time PCRs, and the commercial S. stercoralis real-time PCR; moderate agreement for the in-house T. trichiura real-time PCR and the commercial PCRs targeting A. lumbricoides, N. americanus, and H. nana; fair agreement for the in-house $N$. americanus real-time PCR, and both the in-house and the commercial real-time PCRs targeting E. vermicularis; slight agreement for the in-house $S$. stercoralis real-time PCR; and poor agreement for the in-house A. lumbricoides real-time PCR. Focusing on the quantitative assessment based on $\mathrm{Ct}$-values, there was a variable pattern with significantly lower $\mathrm{Ct}$-values after the nucleic acid extraction without bead-beating for the in-house T. solium real-time PCR, as well as after the bead beating-based nucleic acid extraction for the commercial real-time PCRs targeting A. lumbricoides, N. americanus, $H$. nana, and Schistosoma spp. Again, the significance for Ct-value differences of the inhouse T. solium real-time PCR and the commercial $A$. lumbricoides would be challenged by application of Bonferroni's correction [45]. For all other real-time PCRs, either there was no significant difference or the calculation was impossible because of a lack of positive test results. Relevant sample inhibition was not observed with either extraction scheme. Details are shown in Tables 6-8. 
Table 6. Nucleic acid extraction scheme comparison using the scheme without bead-beating and the scheme with beadbeating on 77 stool samples with the group specific helminth real-time PCRs.

\begin{tabular}{|c|c|c|c|c|c|c|c|}
\hline Genus/Species & Extraction Method & Number $n$ & $\begin{array}{c}\text { Number of } \\
\text { Positives (\%) }\end{array}$ & $p$-Value ${ }^{1}$ & $\begin{array}{c}\text { Ct-Value Mean } \\
( \pm \text { Standard } \\
\text { Deviation SD) }\end{array}$ & $p$-Value $^{2}$ & $\begin{array}{c}\text { Cohen's Kappa } \\
\text { (0.95 Confidence } \\
\text { Interval CI) }\end{array}$ \\
\hline \multirow{2}{*}{$\begin{array}{l}\text { 18S rRNA gene-based } \\
\text { pan-cestode real-time } \\
\text { PCR }\end{array}$} & Without bead beating & 77 & $20(26)$ & \multirow{2}{*}{0.476} & $31.1(3.5)$ & \multirow{2}{*}{0.361} & 0.683 \\
\hline & With bead beating & 77 & $24(31)$ & & $20.0(4.2)$ & & $(0.503,0.863)$ \\
\hline \multirow{2}{*}{$\begin{array}{c}\text { cox gene-based } \\
\text { pan-cestode real-time } \\
\text { PCR }\end{array}$} & Without bead beating & 77 & $25(32)$ & \multirow{2}{*}{0.864} & $35.3(4.5)$ & \multirow{2}{*}{0.093} & 0.853 \\
\hline & With bead beating & 77 & $26(34)$ & & $33.3(4.2)$ & & $(0.729,0.977)$ \\
\hline \multirow{2}{*}{$\begin{array}{c}18 \mathrm{~S} \text { rRNA gene-based } \\
\text { pan-trematode real-time } \\
\text { PCR }\end{array}$} & Without bead beating & 77 & $9(12)$ & \multirow{2}{*}{1} & $28.2(2.3)$ & \multirow{2}{*}{0.588} & 0.874 \\
\hline & With bead beating & 77 & $9(12)$ & & $29.2(4.9)$ & & $(0.703,1)$ \\
\hline \multirow{2}{*}{$\begin{array}{c}\text { 28S rRNA gene-based } \\
\text { pan-trematode real-time } \\
\text { PCR }\end{array}$} & Without bead beating & 77 & $9(12)$ & \multirow{2}{*}{0.797} & $30.4(2.0)$ & \multirow{2}{*}{0.071} & 0.802 \\
\hline & With bead beating & 77 & $8(10)$ & & $28.3(2.4)$ & & $(0.585,1)$ \\
\hline \multirow{2}{*}{$\begin{array}{c}18 \mathrm{~S} \text { rRNA gene-based } \\
\text { pan-nematode real-time } \\
\text { PCR }\end{array}$} & Without bead beating & 77 & $29(38)$ & \multirow{2}{*}{0.621} & $32.1(3.3)$ & \multirow{2}{*}{0.223} & 0.756 \\
\hline & Without bead beating & 77 & $32(42)$ & & $31.1(3.3)$ & & $(0.607,0.905)$ \\
\hline \multirow{2}{*}{$\begin{array}{c}\text { cox gene-based } \\
\text { pan-nematode real-time } \\
\text { PCR }\end{array}$} & Without bead beating & 77 & $18(23)$ & \multirow{2}{*}{0.4623} & $32.4(2.9)$ & \multirow{2}{*}{0.775} & 0.702 \\
\hline & With bead beating & 77 & $22(29)$ & & $32.1(4.5)$ & & $(0.524,0.881)$ \\
\hline
\end{tabular}

${ }^{1}$ Tests of proportions. ${ }^{2}$ Unpaired T-test after nonsignificant testing ( $p$ value $\left.>0.05\right)$ for the equality of variances.

Table 7. Nucleic acid extraction scheme comparison using the scheme without bead-beating and the scheme with beadbeating on 77 stool samples with the genus- and species-specific in-house helminth real-time PCRs.

\begin{tabular}{|c|c|c|c|c|c|c|c|}
\hline Genus/Species & Extraction Method & Number $n$ & $\begin{array}{l}\text { Number of } \\
\text { Positives (\%) }\end{array}$ & $p$-Value ${ }^{1}$ & $\begin{array}{l}\text { Ct-Value Mean } \\
\text { ( } \pm \text { Standard } \\
\text { Deviation SD) }\end{array}$ & $p$-Value ${ }^{2}$ & $\begin{array}{c}\text { Cohen's Kappa } \\
\text { (0.95 Confidence } \\
\text { Interval CI) }\end{array}$ \\
\hline \multirow{2}{*}{$\begin{array}{l}\text { house Ancyclostoma } \\
\text { spp. real-time PCR }\end{array}$} & Without bead beating & 77 & $1(1)$ & \multirow{2}{*}{1} & 30 & \multirow{2}{*}{ n.e. } & \multirow{2}{*}{$1(1,1)$} \\
\hline & With bead beating & 77 & $1(1)$ & & 29 & & \\
\hline \multirow{2}{*}{$\begin{array}{c}\text { in-house Strongyloides } \\
\text { stercoralis real-time } \\
\text { PCR }\end{array}$} & Without bead beating & 77 & $11(14)$ & \multirow{2}{*}{0.113} & $34.2(3.3)$ & \multirow{2}{*}{$0.076 *$} & 0.176 \\
\hline & With bead beating & 77 & $5(6)$ & & $26.0(7.8)$ & & $(-0.113,0.466)$ \\
\hline \multirow{2}{*}{$\begin{array}{c}\text { in-house Necator } \\
\text { americanus real-time } \\
\text { PCR }\end{array}$} & Without bead beating & 77 & $7(9)$ & \multirow{2}{*}{0.006} & $34.4(4.2)$ & \multirow{2}{*}{0.070} & 0.272 \\
\hline & With bead beating & 77 & $20(26)$ & & $31.6(3.2)$ & & $(0.040,0.505)$ \\
\hline \multirow{2}{*}{$\begin{array}{c}\text { in-house Ascaris } \\
\text { lumbricoides real-time } \\
\text { PCR }\end{array}$} & Without bead beating & 77 & $3(4)$ & \multirow{2}{*}{0.043} & $30.3(1.5)$ & \multirow{2}{*}{0.842} & -0.064 \\
\hline & With bead beating & 77 & $10(13)$ & & $30.6(2.1)$ & & $(-0.136,0.008)$ \\
\hline \multirow{2}{*}{$\begin{array}{l}\text { in-house Trichuris } \\
\text { trichiura real-time } \\
\text { PCR }\end{array}$} & Without bead beating & 77 & $14(18)$ & \multirow{2}{*}{0.547} & $29.6(3.2)$ & \multirow{2}{*}{0.330} & 0.476 \\
\hline & With bead beating & 77 & $17(22)$ & & $30.7(2.8)$ & & $(0.233,0.719)$ \\
\hline \multirow{2}{*}{$\begin{array}{l}\text { in-house Taenia solium } \\
\text { real-time PCR }\end{array}$} & Without bead beating & 77 & $2(3)$ & \multirow{2}{*}{0.405} & $27.0(1.4)$ & \multirow{2}{*}{0.043} & 0.655 \\
\hline & With bead beating & 77 & $4(5)$ & & $31.0(1.6)$ & & $(0.211,1)$ \\
\hline \multirow{2}{*}{$\begin{array}{l}\text { in-house Schistosoma } \\
\text { spp. real-time PCR }\end{array}$} & Without bead beating & 77 & $10(13)$ & \multirow{2}{*}{0.616} & $23.3(4.5)$ & \multirow{2}{*}{0.470} & 0.874 \\
\hline & With bead beating & 77 & $8(10)$ & & $21.8(4.3)$ & & $(0.704,1)$ \\
\hline \multirow{2}{*}{$\begin{array}{c}\text { in-house Taenia } \\
\text { saginata real-time } \\
\text { PCR }\end{array}$} & Without bead beating & 77 & $1(1)$ & \multirow{2}{*}{ n.e. } & 18 (n.e.) & \multirow{2}{*}{ n.e. } & \multirow{2}{*}{ n.e. } \\
\hline & With bead beating & 77 & 0 & & n.a. & & \\
\hline \multirow{2}{*}{$\begin{array}{c}\text { in-house Hymenolepis } \\
\text { nana real-time PCR }\end{array}$} & Without bead beating & 77 & $25(32)$ & \multirow{2}{*}{0.864} & $28.9(3.7)$ & \multirow{2}{*}{0.335} & 0.912 \\
\hline & With bead beating & 77 & $26(34)$ & & $27.8(4.4)$ & & $(0.815,1)$ \\
\hline \multirow{2}{*}{$\begin{array}{c}\text { in-house Enterobius } \\
\text { vermicularis real-time } \\
\text { PCR }\end{array}$} & Without bead beating & 77 & $2(3)$ & \multirow{2}{*}{0.146} & $31.5(0.7)$ & \multirow{2}{*}{0.6242} & 0.220 \\
\hline & With bead beating & 77 & $6(8)$ & & $32.7(3.0)$ & & $(-0.176,0.615)$ \\
\hline \multirow{2}{*}{$\begin{array}{c}\text { in-house Schistosoma } \\
\text { mansoni real-time } \\
\text { PCR }\end{array}$} & Without bead beating & 77 & $10(13)$ & 0.374 & $24.4(4.7)$ & $0.094 *$ & 0.705 \\
\hline & With bead beating & 77 & $14(18)$ & $0.3 / 4$ & $29.8(10.0)$ & 0.094 & $(0.486,0.924)$ \\
\hline in-house Schistosoma & Without bead beating & 77 & 0 & ne & n.a. & $n e$ & ne \\
\hline $\begin{array}{l}\text { haematobium real-time } \\
\text { PCR }\end{array}$ & With bead beating & 77 & 0 & n.e. & n.a. & n.e. & n.e. \\
\hline
\end{tabular}

${ }^{1}$ Tests of proportions. ${ }^{2}$ Unpaired T-test after nonsignificant testing ( $p$ value $\left.>0.05\right)$ for the equality of variances. ${ }^{*}$ Unpaired T-test with unequal variances after significant testing for the equality of variances $(p$ value $\leq 0.05)$. n.a. $=$ not applicable. n.e. $=$ nonestimable. 
Table 8. Nucleic acid extraction scheme comparison using the scheme without bead-beating and the scheme with beadbeating on 77 stool samples with the genus- and species-specific commercial Tib MolBiol helminth real-time PCRs.

\begin{tabular}{|c|c|c|c|c|c|c|c|}
\hline Genus/Species & Extraction Method & Number $n$ & $\begin{array}{c}\text { Number of } \\
\text { Positives (\%) }\end{array}$ & $p$-Value ${ }^{1}$ & $\begin{array}{c}\text { Ct-Value Mean } \\
( \pm \text { Standard } \\
\text { Deviation SD) }\end{array}$ & $p$-Value ${ }^{2}$ & $\begin{array}{c}\text { Cohen's Kappa } \\
\text { (0.95 Confidence } \\
\text { Interval CI) }\end{array}$ \\
\hline \multirow{2}{*}{$\begin{array}{c}\text { Tib MolBiol Ascaris } \\
\text { lumbricoides real-time } \\
\text { PCR }\end{array}$} & Without bead beating & 51 & $4(8)$ & \multirow{2}{*}{0.010} & $34.0(2.8)$ & \multirow{2}{*}{0.015} & 0.558 \\
\hline & With bead beating & 77 & $14(18)$ & & $30.2(2.4)$ & & $(0.176,0.941)$ \\
\hline \multirow{2}{*}{$\begin{array}{c}\text { Tib MolBiol } \\
\text { Strongyloides stercoralis } \\
\text { real-time PCR } \\
\end{array}$} & Without bead beating & 51 & $3(6)$ & \multirow{2}{*}{0.508} & $30.7(2.5)$ & \multirow{2}{*}{0.746} & 0.847 \\
\hline & With bead beating & 77 & $7(9)$ & & $29.3(6.7)$ & & $(0.553,1)$ \\
\hline \multirow{2}{*}{$\begin{array}{l}\text { Tib MolBiol Necator } \\
\text { americanus real-time } \\
\text { PCR }\end{array}$} & Without bead beating & 51 & $9(18)$ & \multirow{2}{*}{0.045} & $34.2(3.0)$ & \multirow{2}{*}{0.001} & 0.582 \\
\hline & With bead beating & 77 & $26(34)$ & & $29.5(3.5)$ & & $(0.308,0.857)$ \\
\hline \multirow{2}{*}{$\begin{array}{l}\text { Tib MolBiol } \\
\text { Ancylostoma spp. } \\
\text { real-time PCR }\end{array}$} & Without bead beating & 51 & $1(2)$ & \multirow{2}{*}{0.7675} & 33.00 & \multirow{2}{*}{ n.e. } & 1 \\
\hline & With bead beating & 77 & $1(1)$ & & 32.00 & & $(1,1)$ \\
\hline \multirow{2}{*}{$\begin{array}{c}\text { Tib MolBiol } \\
\text { Hymenolepis nana } \\
\text { real-time PCR }\end{array}$} & Without bead beating & 45 & $7(16)$ & \multirow{2}{*}{0.041} & $32.3(2.4)$ & \multirow{2}{*}{0.003} & 0.588 \\
\hline & With bead beating & 77 & $25(32)$ & & $26.6(4.4)$ & & $(0.300,0.877)$ \\
\hline \multirow{2}{*}{$\begin{array}{l}\text { Tib MolBiol Enterobius } \\
\text { vermicularis real-time } \\
\text { PCR }\end{array}$} & Without bead beating & 45 & $2(4)$ & \multirow{2}{*}{0.063} & $32.5(0.7)$ & \multirow{2}{*}{0.8012} & 0.237 \\
\hline & With bead beating & 77 & $12(16)$ & & $31.8(3.5)$ & & $(-0.206,0.680)$ \\
\hline \multirow{2}{*}{$\begin{array}{l}\text { Tib MolBiol } \\
\text { Schistosoma spp. } \\
\text { real-time PCR }\end{array}$} & Without bead beating & 45 & $9(20)$ & \multirow{2}{*}{0.085} & $29.3(2.5)$ & \multirow{2}{*}{$<0.001$} & 0.848 \\
\hline & With bead beating & 77 & $7(9)$ & & $22.1(1.5)$ & & $(0.646,1)$ \\
\hline
\end{tabular}

${ }^{1}$ Tests of proportions. ${ }^{2}$ Unpaired T-test after nonsignificant testing $(p$ value $>0.05)$ for the equality of variances. n.e. $=$ nonestimable.

The low number of external quality assessment scheme samples [40] did not allow an independent statistical assessment for those samples. With those samples, however, positive real-time PCR signals in case of discordant results were observed after beadbeating-based extraction only. A small proportion of $\mathrm{Ct}$-values showed identical results irrespective of the extraction scheme within a $\pm 1 \mathrm{Ct}$ step range. A larger proportion showed lower $\mathrm{Ct}$ values after bead-beating-based extraction compared with the QiaAMP DNA Stool Mini Kit-based extraction. Convincing significance, however, could not be shown for differences in the Ct-value-distribution between the quality assessment scheme samples and the other samples. In detail, the distribution of the $\mathrm{Ct}$ values for all real-time PCRs with at least three recorded $\mathrm{Ct}$ values per sample group was evaluated. The KolmogorovSmirnov test was used, which tests the empirical distribution in the sample groups for difference. The null hypothesis was that the distribution of the $\mathrm{Ct}$ values of both samples was identical. The required level of significance was 0.05 . For seven real-time PCR assays for which the abovementioned conditions were fulfilled, $p$ values were $0.046,0.518,0.514$, $0.080,0.426,0.431$, and 0.334 , respectively.

\subsection{Comparison of Qualitative and Quantitative Results of Nonhelminth Real-Time PCRs after the Two Compared Nucleic Acid Extraction Procedures}

Focusing on the qualitative results of the eight in-house real-time PCRs targeting bacteria and protozoa in 67 samples, significantly more positive results were found for Shigella spp./EIEC after bead beating-based nucleic acid extraction. No significant difference was detectable for any other parameter. Again, there was a wide spectrum of agreement, with substantial agreement for the in-house real-time PCRs targeting Salmonella spp., C. jejuni, and G. duodenalis and slight agreement for Shigella spp./EIEC and Cyclospora spp. For the other parameters, numbers of positive results were insufficient to allow the calculation of Cohen's kappa. Significant difference in recorded Ct-values were never observed for the nonhelminth parameters. Details are shown in Table 9.

\subsection{Photometric Assessment of DNA Concentrations within the Samples}

After performing all real-time PCR assessments, sufficient residual sample volumes for photometric DNA quantification were available for 39 nucleic acid extractions without bead beating and for 77 nucleic acid extractions with bead beating as well as for 96 historic samples and 20 fresh external quality assessment scheme samples, respectively. There was no statistically significant difference between the DNA-concentrations in comparison of 
fresh external quality assessment scheme samples and historic samples (mean of $20.0 \mathrm{ng} / \mu \mathrm{L}$ for fresh quality control assessment scheme samples, mean of $23.0 \mathrm{ng} / \mu \mathrm{L}$ for historic samples, $p=0.99$ ), while bead beating-based nucleic acid extraction (mean of $16.2 \mathrm{ng} / \mu \mathrm{L}$ ) yielded about half as much DNA as nuclei acid extraction without bead beating (mean of $35.0 \mathrm{ng} / \mu \mathrm{L})(p=0.0003) . p$ values were calculated using the Mann-Whitney U-test.

Table 9. Nucleic acid extraction scheme comparison using the scheme without bead-beating and the scheme with beadbeating with real-time PCRs for enteropathogenic bacteria and enteric protozoa on 67 stool samples.

\begin{tabular}{|c|c|c|c|c|c|c|c|}
\hline Genus/Species & Extraction Method ${ }^{\#}$ & Number $n$ & $\begin{array}{l}\text { Number of } \\
\text { Positives (\%) }\end{array}$ & $p$-Value ${ }^{1}$ & $\begin{array}{l}\text { Ct-Value Mean } \\
\text { ( } \pm \text { Standard } \\
\text { Deviation SD) }\end{array}$ & $p$-Value ${ }^{2}$ & $\begin{array}{c}\text { Cohen's Kappa } \\
\text { (0.95 Confidence } \\
\text { Interval CI) }\end{array}$ \\
\hline \multirow{2}{*}{$\begin{array}{l}\text { In-house Salmonella spp. } \\
\text { real-time PCR }\end{array}$} & Without bead beating & 67 & $3(4)$ & \multirow{2}{*}{0.649} & $31.3(2.1)$ & \multirow{2}{*}{0.334} & 0.793 \\
\hline & With bead beating & 67 & $2(3)$ & & $29.5(0.7)$ & & $(0.398,1)$ \\
\hline \multirow{2}{*}{$\begin{array}{l}\text { In-house Yersinia spp. real-time } \\
\text { PCR }\end{array}$} & Without bead beating & 67 & 0 & \multirow{2}{*}{ n.e. } & n.a. & \multirow{2}{*}{ n.e. } & \multirow{2}{*}{ n.e. } \\
\hline & With bead beating & 67 & 0 & & n.a. & & \\
\hline \multirow{2}{*}{$\begin{array}{c}\text { In-house Campylobacter jejuni } \\
\text { real-time PCR }\end{array}$} & Without bead beating & 67 & $12(18)$ & \multirow{2}{*}{0.819} & $25.8(3.2)$ & \multirow{2}{*}{0.578} & 0.633 \\
\hline & With bead beating & 67 & $11(16)$ & & $26.5(3.6)$ & & $(0.384,0.882)$ \\
\hline \multirow{2}{*}{$\begin{array}{c}\text { In-house Shigella } \\
\text { spp./enteroinvasive Escherichia } \\
\text { coli real-time PCR }\end{array}$} & Without bead beating & 67 & $2(3)$ & \multirow{2}{*}{$<0.001$} & $31.0(1.4)$ & \multirow{2}{*}{0.821} & 0.179 \\
\hline & With bead beating & 67 & $16(24)$ & & $31.6(3.7)$ & & $(-0.040,0.397)$ \\
\hline \multirow{2}{*}{$\begin{array}{l}\text { In-house E. histolytica } \\
\text { real-time PCR }\end{array}$} & Without bead beating & 67 & 0 & \multirow{2}{*}{ n.e. } & n.a. & \multirow{2}{*}{ n.e. } & \multirow{2}{*}{ n.e. } \\
\hline & With bead beating & 67 & $1(1)$ & & 33 (n.e.) & & \\
\hline \multirow{2}{*}{$\begin{array}{l}\text { In-house Cyclospora spp. } \\
\text { real-time PCR }\end{array}$} & Without bead beating & 67 & $6(9)$ & \multirow{2}{*}{0.572} & $30.8(2.9)$ & \multirow{2}{*}{$0.059 *$} & 0.045 \\
\hline & With bead beating & 67 & $8(12)$ & & $33.8(0.9)$ & & $(-0.223,0.313)$ \\
\hline \multirow{2}{*}{$\begin{array}{l}\text { In-house Giardia duodenalis } \\
\text { real-time PCR }\end{array}$} & Without bead beating & 67 & $19(28)$ & \multirow{2}{*}{1} & $28.7(3.9)$ & \multirow{2}{*}{0.194} & 0.706 \\
\hline & With bead beating & 67 & $19(28)$ & & $27.1(3.7)$ & & $(0.517,0.895)$ \\
\hline \multirow{2}{*}{$\begin{array}{l}\text { In-house Cryptosporidium spp. } \\
\text { real-time PCR }\end{array}$} & Without bead beating & 67 & 0 & \multirow{2}{*}{ n.e. } & n.a. & \multirow{2}{*}{ n.e. } & \multirow{2}{*}{ n.e. } \\
\hline & With bead beating & 67 & $1(1)$ & & 33.0 (n.e.) & & \\
\hline
\end{tabular}

${ }^{1}$ Tests of proportions. ${ }^{2}$ Unpaired T-test after nonsignificant testing $(p$ value $>0.05)$ for the equality of variances. ${ }^{*}$ Unpaired T-test with unequal variances after significant testing for the equality of variances $(p$ value $\leq 0.05)$. n.a. $=$ not applicable. n.e. $=$ nonestimable.

\section{Discussion}

The study was performed to compare performance characteristics of two different nucleic extraction procedures for diagnostic real-time PCR approaches targeting helminths. A number of genus- and species-specific in-house helminth real-time PCR approaches $[28,42,43]$ supplemented by some commercial assays [28] were applied. Among the in-house assays were both previously described $[29,30]$ and newly introduced group-specific helminth real-time PCRs targeting nematodes, trematodes, and cestodes. With both microscopically positive and negative samples (details are shown in Tables 4 and 5 and Appendix A), these group-specific real-time PCRs showed imperfect sensitivity and specificity, predominantly with sensitivity issues. This makes them particularly interesting for potential improvement following the comparison of nucleic acid extraction procedures. Due to the low number of available microscopically positive samples and due to the detection limits of microscopy, recorded sensitivity and specificity values have to be interpreted with care. Accordingly, the study was focused on the comparison of sensitivity as affected by the mode of nucleic acid extraction only.

As well as a standard approach for nucleic acid extraction from stool samples, a more sophisticated approach including bead-beating and proteinase K-digestion was applied as suggested in a recent publication [33]. Most interestingly, however, in the present study this harsher extraction scheme was not generally superior. Instead, there were different reaction patterns depending on the real-time PCR assay used. In detail, benefits were seen for robust eggs of $A$. lumbricoides but also for fragile $N$. americanus eggs. For T. trichiura — a nematode with very robust eggs [33] — effects were less obvious, which is in contrast with previous works $[33,34]$. Nonsignificant tendencies of stronger influence 
of the bead-beating-based extraction scheme in standardized external quality assessment scheme samples - for which an independent statistical evaluation had low power due to the low number of available specimens-suggest likely but nonsignificant effects of sample age of the assessed sample collection. However, even within the external quality assessment scheme specimens, several samples showed identical Ct-values irrespective of the chosen nucleic acid extraction procedures. In contrast, considerable shifts of several decadic logarithmic steps were observed for other samples. The reasons for this observed inconsistency are unclear.

Of note, however, considerable differences between real-time PCR results following the different extraction schemes suggest detections at the threshold of diagnostic sensitivity. Close to the detection threshold, positive results necessarily tend to become stochastic phenomena. There was a marked dominance of lower Ct-values after the bead-beatingbased extraction scheme for the commercial test assays compared to the in-house assays. The reasons for this remain unclear: different sensitivity of the assays might theoretically play a role here. However, a previous assessment [28] did not suggest marked differences between the in-house and the commercial real-time PCR approaches. At least for the Ct-values with the commercial real-time PCR assays, superiority of the bead beating-based extraction can be considered likely. In contrast, the results are less conclusive for the in-house approaches. For example, when looking specifically at the samples from the quality control assessment scheme (data not shown), the in-house assays also showed more positives with considerably lower Ct-values. An exception was T. trichiura, for which the recorded Ct-values were similar. However, those differences are mostly lost when assessing the whole sample collection.

Interestingly, however, not even the commercial assay showed unambiguous difference for the robust eggs of T. trichiura, which is in contrast to previous results [33,34]. As differences in results depending on the producer of the applied beads are known from previous assessments [33], minor differences in the production and resulting microstructure of the garnet beads might be a potential reason for this striking difference. This is an issue that might make the standardization of bead-beating extraction for helminth real-time PCRs challenging if suppliers are not interchangeable. The results with the external quality assessment scheme samples (data not shown) at least suggest a tendency toward more positive results and lower Ct-values after bead-beating-based extraction. So, the particular situation of working with historical samples may provide an explanation for this observed contradiction with previous studies as well, although the precise history of the external quality assessment scheme samples was unknown as well.

Considerable, target-dependent, variability in agreement of the results following the different nucleic acid extraction strategies was observed for the real-time PCRs with nonhelminth targets. Significantly more detection of Shigella spp./EIEC after the beadbased nucleic acid extraction strategy was counteracted by only slight agreement, so this result is difficult in interpret. Significant differences in the measured Ct-values were never observed, however.

The present study has a number of limitations. First, the samples that were used for the extraction comparison were not assessed microscopically. So, definite microscopic proof of the presence of pathogens was not feasible. Secondly, due to the rarity of occurrence of helminth-positive sample materials, the overall numbers of samples analyzed was low. Thirdly, and for the same reasons, the ages of the samples used varied between a few months and about 10 years, although frozen storage at $-80{ }^{\circ} \mathrm{C}$ makes quantitatively relevant nucleic acid degradation less likely. Fourthly, there was no homogenization step of the initially provided stool samples, making uneven distributions of parasites in stool likely. Fifthly, real-time PCRs after different extractions were not consistently performed in parallel in the same run, and low volumes of residual sample materials did not allow repeated testing. So, the comparison indeed reflects routine-like conditions. Sixthly, due to limited available sample volumes, photometric assessments of the DNA concentration within the eluates could be performed only for a subset of the samples included in the 
study. Accordingly, effects to varying DNA concentrations after the different extraction schemes could not be analyzed for all samples. However, an exploratory investigation with the available residual sample materials did not indicate significant influence of sample age on measured DNA concentrations. Although the extraction scheme without bead beating yielded about twice as much DNA compared to the extraction scheme with bead beating, an average factor of two will only have minor influence on measured $\mathrm{Ct}$ values in real-time PCR. Seventhly, individual fecal samples contain variable concentrations of inhibitory components [46], potentially resulting in heterogeneity to the set of samples and the outcome of real-time PCR testing in case of uneven distribution within the sample due to the missing homogenization step. However, inhibition control real-time PCR based on a Phocid Herpes DNA sequence as described elsewhere [47] did not suggest relevant inhibition issues (data not shown). Eighthly, only frozen samples with potential effects of freezing on DNA release from helminth cells could be assessed. Ninthly, only human stool samples were included in the assessment. The analysis of other sample materials would have been interesting as well but was beyond the scope of this assessment.

In spite of these limitations, the assessment indicated that the harsh nucleic acid extraction scheme could indeed provide considerable shifts in Ct-values for several samples and also provided tendencies of higher sensitivity. However, the observation of $\mathrm{Ct}$-value shifts is inconsistent. Especially with historic sample materials, which may play a role in retrospective epidemiological assessments rather than for routine diagnostic applications, the beneficial effects of the harsh extraction approach are less obvious than in previous reports assessing fresh samples. Nevertheless, early international external quality assessment trials [40] have suggested the applicability of real-time PCR for the diagnosis of helminths. Accordingly, broader application of such strategies is to be expected in the future, making studies on further optimization of pre-analytic conditions desirable.

\section{Conclusions}

This nucleic acid extraction comparison indicated inconsistent effects of the analyzed harsh nucleic acid extraction scheme on helminth real-time PCRs. There were only tendencies for beneficial effects of bead-beating-based extraction as observed with the samples from the external quality assessment scheme. If complex nucleic acid extraction schemes are infeasible for logistical reasons in diagnostic or study settings, sensitivity constraints may result. With historical samples in retrospective assessments, any beneficial effects of harsh nucleic acid extraction may be less pronounced than observed with fresh sample materials in the diagnostic routine in previous studies.

Author Contributions: Conceptualization, H.F. and U.L.; methodology, T.H., A.H., J.J.V., G.L., O.L., and H.F.; software, T.H. and A.H.; validation, T.H. and H.F.; formal analysis, A.H.; investigation, T.H. and H.F.; resources, C.S., S.K., D.D., J.M., H.F. and U.L.; data curation, T.H., A.H. and H.F.; writingoriginal draft preparation, H.F. and A.H.; writing-review and editing, all authors; visualization, not applicable; supervision, H.F.; project administration, H.F.; funding acquisition, H.F. and U.L. All authors have read and agreed to the published version of the manuscript.

Funding: The experiments were funded by grant 36K2-S-45 1922 "Evaluation and optimization of molecular diagnostic tests for tropical parasitic diseases for surveillance and risk assessment purposes in tropical deployment settings-a German-French cooperation project between the German Armed Forces Hospital Hamburg and the Military Hospital Laveran, Marseille" of the German Ministry of Defense (MoD) awarded to Hagen Frickmann. We acknowledge support by the Open Access Publication Funds of the University of Göttingen. The sponsors did not have any role in the collection, analysis, or interpretation of data, in the writing of the report, or in the decision to submit the article for publication.

Institutional Review Board Statement: Ethical clearance, provided for blinded use of residual materials for test comparison and evaluation purposes, was granted on 11 March 2019 by the ethics committee of the Medical Association of Hamburg, Germany (registration number WF-011/19) in line with national laws without requirement for informed consent. The authors assert that all procedures contributing to this work comply with the Helsinki Declaration of 1975, as revised in 2008. 
Informed Consent Statement: Not applicable.

Data Availability Statement: All relevant data are provided within the manuscripts and its tables. Raw data can be made available on reasonable request.

Acknowledgments: Annett Michel and Simone Priesnitz are gratefully acknowledged for excellent technical assistance. Christoph Grevelding, Minka Breloer, and Egbert Tannich are acknowledged for providing individual reference samples for the assessments of applied group specific helminth real-time PCRs.

Conflicts of Interest: G.L., and O.L. are representatives of the company TIB MOLBIOL. The funders had no role in the design of the study; in the collection, analyses, or interpretation of data; in the writing of the manuscript, or in the decision to publish the results.

\section{Appendix A}

Table A1. Details of the assessment of group specific helminth real-time PCRs with residual DNAs of stool samples tested positive with genus- or specific helminth real-time PCR (see also Table 4).

\begin{tabular}{|c|c|c|c|c|c|c|c|c|}
\hline $\begin{array}{l}\text { Sample } \\
\text { Number }\end{array}$ & $\begin{array}{c}\text { Species/Genus According to } \\
\text { in-House Real-Time PCR (Ct } \\
\text { Value) }\end{array}$ & $\begin{array}{l}\text { Expected } \\
\text { Consensus } \\
\text { Real-Time } \\
\text { PCR Result }\end{array}$ & $\begin{array}{l}\text { Ct Values of } \\
\text { Nematode } \\
\text { Consensus } \\
\text { Real-Time } \\
\text { PCR } 2\end{array}$ & $\begin{array}{c}\text { Ct Values of } \\
\text { Trematode } \\
\text { Consensus } \\
\text { Real-Time } \\
\text { PCR } 2\end{array}$ & $\begin{array}{l}\text { Ct Values of } \\
\text { Cestode } \\
\text { Consensus } \\
\text { Real-Time } \\
\text { PCR } 2\end{array}$ & $\begin{array}{c}\text { Ct Values of } \\
\text { Nematode } \\
\text { Consensus } \\
\text { Real-Time } \\
\text { PCR } 1\end{array}$ & $\begin{array}{l}\text { Ct Value of } \\
\text { Trematode } \\
\text { Consensus } \\
\text { Real-Time } \\
\text { PCR } 1\end{array}$ & $\begin{array}{l}\text { Ct Value of } \\
\text { Cestode } \\
\text { Consensus } \\
\text { Real-Time } \\
\text { PCR } 1\end{array}$ \\
\hline 1 & Hymenolepis nana (25) & Cestode & & & 29 & & & 33 \\
\hline 2 & Hymenolepis nana (27) & Cestode & & & & & & 36 \\
\hline 3 & Hymenolepis nana (29) & Cestode & & & 32 & & & 34 \\
\hline 4 & Hymenolepis nana (27) & Cestode & 30 & & 30 & & & 35 \\
\hline 5 & Hymenolepis nana (32) & Cestode & & & 33 & & & 33 \\
\hline 6 & Hymenolepis nana (23) & Cestode & & & 27 & & & 29 \\
\hline 7 & Hymenolepis nana (33) & Cestode & & & 36 & & & 39 \\
\hline 8 & Trichuris trichiura (30) & Nematode & 32 & & & & & \\
\hline 9 & Hymenolepis nana (29) & Cestode & & & 31 & & & 34 \\
\hline 10 & Trichuris trichiura (33) & Nematode & 36 & & & & & \\
\hline 11 & Hymenolepis nana (29) & Cestode & & & 32 & & & 36 \\
\hline 12 & Trichuris trichiura (24) & Nematode & 30 & & & & & \\
\hline 13 & Hymenolepis nana (29) & Cestode & & & & & & 33 \\
\hline 14 & Hymenolepis nana (29) & Cestode & & & 33 & & & 34 \\
\hline 15 & Schistosoma spp. (34) & Trematode & & & & & & \\
\hline 16 & Ascaris lumbricoides (29) & Nematode & & & & & & \\
\hline 17 & Trichuris trichiura (32) & Nematode & 32 & & & 32 & & \\
\hline 18 & Trichuris trichiura (26) & Nematode & 30 & & & & & \\
\hline 19 & Taenia solium (26) & Cestode & & & 30 & & & 29 \\
\hline 20 & Necator americanus (36) & Nematode & 35 & & & & & \\
\hline 21 & Trichuris trichiura (27) & Nematode & & & & 34 & & \\
\hline 22 & $\begin{array}{l}\text { Hymenolepis nana (29), } \\
\text { Enterobius vermicularis (36) }\end{array}$ & $\begin{array}{l}\text { Nematode, } \\
\text { cestode }\end{array}$ & 29 & & 32 & & & 32 \\
\hline 23 & Necator americanus (34) & Nematode & 29 & & & 31 & & 33 \\
\hline 24 & Ascaris lumbricoides (30) & Nematode & 31 & & & 35 & & \\
\hline 25 & Hymenolepis nana (30) & Cestode & 33 & & & & & 39 \\
\hline 26 & Trichuris trichiura (29) & Nematode & 32 & & & & & \\
\hline 27 & Necator americanus (36) & Nematode & 30 & & & 29 & & \\
\hline 28 & Ascaris lumbricoides (32) & Nematode & 41 & & & 32 & & \\
\hline 29 & Hymenolepis nana (25) & Cestode & 37 & & 30 & & & 33 \\
\hline 30 & Hymenolepis nana (32) & Cestode & 31 & & & 30 & & 40 \\
\hline 31 & Hymenolepis nana (31) & Cestode & & & & & & 38 \\
\hline 32 & Hymenolepis nana (29) & Cestode & 30 & & 35 & & & 38 \\
\hline 33 & Enterobius vermicularis (32) & Nematode & 32 & & & 29 & & \\
\hline 34 & Taenia solium (28) & Cestode & 28 & & & 34 & & \\
\hline 35 & $\begin{array}{l}\text { Strongyloides stercoralis (31), } \\
\text { Necator americanus (37) }\end{array}$ & Nematode & 32 & & & 42 & & \\
\hline 36 & Trichuris trichiura (27) & Nematode & 32 & & & 32 & & \\
\hline 37 & Trichuris trichiura (25) & Nematode & 32 & & & 32 & & \\
\hline 38 & Ascaris lumbricoides (28) & Nematode & & & & & & \\
\hline 39 & Hymenolepis nana (29) & Cestode & 31 & & & & & \\
\hline 40 & Trichuris trichiura (28) & Nematode & 32 & & & 31 & & \\
\hline 41 & Trichuris trichiura (27) & Nematode & 32 & & & 30 & & \\
\hline 42 & Trichuris trichiura (28) & Nematode & 31 & & & 35 & & 35 \\
\hline 43 & Hymenolepis nana (25) & Cestode & 31 & & & 35 & & 35 \\
\hline 44 & Hymenolepis nana (30) & Cestode & 28 & & 28 & 34 & & \\
\hline 45 & Trichuris trichiura (31) & Nematode & & & & & & \\
\hline 46 & Hymenolepis nana (17) & Cestode & & & 20 & & & 29 \\
\hline 47 & Taenia saginata (18) & Cestode & & & & & & \\
\hline 48 & Enterobius vermicularis (33) & Nematode & & & & & & \\
\hline 49 & Strongyloides stercoralis (37) & Nematode & & & & & & \\
\hline 50 & Necator americanus (26) & Nematode & 29 & & & & & \\
\hline
\end{tabular}


Table A1. Cont.

\begin{tabular}{|c|c|c|c|c|c|c|c|c|}
\hline $\begin{array}{l}\text { Sample } \\
\text { Number }\end{array}$ & $\begin{array}{c}\text { Species/Genus According to } \\
\text { in-House Real-Time PCR (Ct } \\
\text { Value) }\end{array}$ & $\begin{array}{c}\text { Expected } \\
\text { Consensus } \\
\text { Real-Time } \\
\text { PCR Result }\end{array}$ & $\begin{array}{l}\text { Ct Values of } \\
\text { Nematode } \\
\text { Consensus } \\
\text { Real-Time } \\
\text { PCR } 2\end{array}$ & $\begin{array}{l}\text { Ct Values of } \\
\text { Trematode } \\
\text { Consensus } \\
\text { Real-Time } \\
\text { PCR } 2\end{array}$ & $\begin{array}{l}\text { Ct Values of } \\
\text { Cestode } \\
\text { Consensus } \\
\text { Real-Time } \\
\text { PCR } 2\end{array}$ & $\begin{array}{c}\text { Ct Values of } \\
\text { Nematode } \\
\text { Consensus } \\
\text { Real-Time } \\
\text { PCR } 1\end{array}$ & $\begin{array}{l}\text { Ct Value of } \\
\text { Trematode } \\
\text { Consensus } \\
\text { Real-Time } \\
\text { PCR } 1\end{array}$ & $\begin{array}{l}\text { Ct Value of } \\
\text { Cestode } \\
\text { Consensus } \\
\text { Real-Time } \\
\text { PCR } 1\end{array}$ \\
\hline 51 & Necator americanus (38) & Nematode & & & & & & \\
\hline 52 & Strongyloides stercoralis (33) & Nematode & & & & & & \\
\hline 53 & Strongyloides stercoralis (41) & Nematode & & & & & & \\
\hline 54 & Strongyloides stercoralis (35) & Nematode & & & & 39 & & \\
\hline 55 & Hymenolepis nana (30) & Cestode & & & & 36 & & \\
\hline 56 & Strongyloides stercoralis (36) & Nematode & & & & & & \\
\hline 57 & Enterobius vermicularis (31) & Nematode & & & & & & \\
\hline 58 & Strongyloides stercoralis (34) & Nematode & & & & & & \\
\hline 59 & Strongyloides stercoralis (36) & Nematode & & & & & & \\
\hline 60 & Strongyloides stercoralis (37) & Nematode & & & & & & \\
\hline 61 & Strongyloides stercoralis (36) & Nematode & & & & & & \\
\hline 62 & $\begin{array}{l}\text { Schistosoma spp. (20), } \\
\text { Strongyloides stercoralis (40) }\end{array}$ & $\begin{array}{l}\text { Trematode, } \\
\text { nematode }\end{array}$ & & 26 & & & 27 & \\
\hline 63 & Schistosoma spp. (26) & Trematode & & 33 & & & 35 & \\
\hline 64 & Schistosoma spp. (22) & Trematode & & 30 & & & 30 & \\
\hline 65 & Hymenolepis nana (30) & Cestode & & & 32 & & & 40 \\
\hline 66 & Schistosoma spp. (17) & Trematode & & 24 & & & 28 & \\
\hline 67 & Schistosoma spp. (28) & Trematode & & 35 & & & & \\
\hline 68 & Schistosoma spp. (23) & Trematode & & 28 & & & 31 & \\
\hline 69 & Hymenolepis nana (27) & Cestode & & & 30 & & & 36 \\
\hline 70 & Strongyloides stercoralis (36) & Nematode & & & & & & \\
\hline 71 & Hymenolepis nana (30) & Cestode & & & & & & \\
\hline 72 & Hymenolepis nana (29) & Cestode & & & 32 & & & 37 \\
\hline 73 & Schistosoma spp. (23) & Trematode & & 29 & & & 34 & \\
\hline 74 & Schistosoma spp. (20) & Trematode & & 26 & & & 29 & \\
\hline 75 & Schistosoma spp. (22) & Trematode & & 27 & 36 & & 29 & 44 \\
\hline 76 & Hymenolepis nana (31) & Cestode & & & & & & 43 \\
\hline 77 & Hymenolepis nana (29) & Cestode & & & 32 & & & 38 \\
\hline 78 & Hymenolepis nana (32) & Cestode & & & 34 & & & 43 \\
\hline 79 & Hymenolepis nana (35) & Cestode & & & 36 & & & 37 \\
\hline 80 & Schistosoma spp. (26) & Trematode & & 31 & & & 33 & \\
\hline 81 & Hymenolepis nana (32) & Cestode & & & 32 & & & 35 \\
\hline 82 & Strongyloides stercoralis (35) & Nematode & & & & & & \\
\hline 83 & Hymenolepis nana (31) & Cestode & & & 34 & & & \\
\hline 84 & Schistosoma spp. (21) & Trematode & & & 28 & & & 29 \\
\hline 85 & Strongyloides stercoralis (34) & Nematode & & & & & & \\
\hline 86 & Trichuris trichiura (34) & Nematode & & & & & & \\
\hline 87 & Strongyloides stercoralis (33) & Nematode & & & & & & 47 \\
\hline 88 & Strongyloides stercoralis (31) & Nematode & & & 37 & & & \\
\hline 89 & Strongyloides stercoralis (32) & Nematode & & & & & & \\
\hline 90 & Necator americanus (25) & Nematode & & 26 & & 30 & & \\
\hline 91 & Strongyloides stercoralis (31) & Nematode & & & & & & \\
\hline 92 & Enterobius vermicularis (36) & Nematode & & & & & & \\
\hline 93 & Schistosoma spp. (32) & Trematode & & & & & & \\
\hline 94 & Strongyloides stercoralis (32) & Nematode & & & & & & \\
\hline 95 & Ascaris lumbricoides (39) & Nematode & & & & & & \\
\hline 96 & Strongyloides stercoralis (32) & Nematode & & & & & & 45 \\
\hline
\end{tabular}

Potentially falsely positive results as compared with genus- or species-specific real-time PCR are highlighted in gray, hypothetically falsely negative results as compared with genus- or species-specific real-time PCR are indicated by a black field.

\section{References}

1. Hotez, P.J.; Brindley, P.J.; Bethony, J.M.; King, C.H.; Pearce, E.J.; Jacobson, J. Helminth infections: The great neglected tropical diseases. J. Clin. Investig. 2008, 118, 1311-1321. [CrossRef] [PubMed]

2. Brooker, S. Estimating the global distribution and disease burden of intestinal nematode infections: Adding up the numbers-A review. Int. J. Parasitol. 2010, 40, 1137-1144. [CrossRef]

3. Taylor, M.J.; Hoerauf, A.; Bockarie, M. Lymphatic filariasis and onchocerciasis. Lancet 2010, 376, 1175-1185. [CrossRef]

4. Chammartin, F.; Scholte, R.G.C.; Guimarães, L.H.; Tanner, M.; Utzinger, J.; Vounatsou, P. Soil-transmitted helminth infection in South America: A systematic review and geostatistical meta-analysis. Lancet Infect. Dis. 2013, 13, 507-518. [CrossRef]

5. Pullan, R.L.; Smith, J.L.; Jasrasaria, R.; Brooker, S. Global numbers of infection and disease burden of soil transmitted helminth infections in 2010. Parasites Vectors 2014, 7, 37. [CrossRef]

6. Torgerson, P.R.; Abdybekova, A.M.; Minbaeva, G.; Shapiyeva, Z.; Thomas, L.F.; Dermauw, V.; Devleesschauwer, B.; Gabriël, S.; Dorny, P.; Braae, U.C.; et al. Epidemiology of Taenia saginata taeniosis/cysticercosis: A sys-tematic review of the distribution in central and western Asia and the Caucasus. Parasit Vectors 2019, 12, 175. [CrossRef]

7. Torgerson, P.R.; Devleesschauwer, B.; Praet, N.; Speybroeck, N.; Willingham, A.L.; Kasuga, F.; Rokni, M.B.; Zhou, X.-N.; Fèvre, E.M.; Sripa, B.; et al. World Health Organization Estimates of the Global and Regional Disease Burden of 11 Foodborne Parasitic Diseases, 2010: A Data Synthesis. PLoS Med. 2015, 12, e1001920. [CrossRef] [PubMed]

8. Qian, M.-B.; Utzinger, J.; Keiser, J.; Zhou, X.-N. Clonorchiasis. Lancet 2016, 387, 800-810. [CrossRef] 
9. Braae, U.C.; Thomas, L.F.; Robertson, L.J.; Dermauw, V.; Dorny, P.; Willingham, A.L.; Saratsis, A.; Devleesschauwer, B. Epidemiology of Taenia saginata taeniosis/cysticercosis: A systematic review of the distribution in the Americas. Parasites Vectors 2018, 11, 518. [CrossRef] [PubMed]

10. Idris, O.A.; Wintola, O.A.; Afolayan, A.J. Helminthiases; prevalence, transmission, host-parasite interactions, resistance to common synthetic drugs and treatment. Heliyon 2019, 5, e01161. [CrossRef] [PubMed]

11. Saratsis, A.; Sotiraki, S.; Braae, U.C.; Devleesschauwer, B.; Dermauw, V.; Eichenberger, R.M.; Thomas, L.F.; Bobić, B.; Dorny, P.; Gabriël, S.; et al. Epidemiology of Taenia saginata taeniosis/cysticercosis: A systematic review of the distribution in the Middle East and North Africa. Parasites Vectors 2019, 12, 113. [CrossRef]

12. Verweij, J.J.; Brienen, E.A.T.; Ziem, J.; Polderman, A.M.; Yelifari, L.; Van Lieshout, L. Simultaneous Detection and Quantification of Ancylostoma duodenale, Necator americanus, and Oesophagostomum bifurcum in Fecal Samples Using Multiplex Real-Time PCR. Am. J. Trop. Med. Hyg. 2007, 77, 685-690. [CrossRef] [PubMed]

13. Verweij, J.J.; Canales, M.; Polman, K.; Ziem, J.; Brienen, E.A.; Polderman, A.M.; Van Lieshout, L. Molecular diagnosis of Strongyloides stercoralis in faecal samples using real-time PCR. Trans. R. Soc. Trop. Med. Hyg. 2009, 103, 342-346. [CrossRef]

14. Obeng, B.; Aryeetey, Y.; De Dood, C.J.; Amoah, A.; Larbi, I.; Deelder, A.; Yazdanbakhsh, M.; Hartgers, F.; Boakye, D.; Verweij, J.; et al. Application of a circulating-cathodic-antigen (CCA) strip test and real-time PCR, in comparison with microscopy, for the detection ofSchistosoma haematobiumin urine samples from Ghana. Ann. Trop. Med. Parasitol. 2008, 102, 625-633. [CrossRef]

15. Hove, R.T.; Verweij, J.J.; Vereecken, K.; Polman, K.; Dieye, L.; Van Lieshout, L. Multiplex real-time PCR for the detection and quantification of Schistosoma mansoni and S. haematobium infection in stool samples collected in northern Senegal. Trans. R. Soc. Trop. Med. Hyg. 2008, 102, 179-185. [CrossRef]

16. Hove, R.J.T.; Van Esbroeck, M.; Vervoort, T.; Ende, J.V.D.; Van Lieshout, L.; Verweij, J.J. Molecular diagnostics of intestinal parasites in returning travellers. Eur. J. Clin. Microbiol. Infect. Dis. 2009, 28, 1045-1053. [CrossRef]

17. Kim, E.M.; Verweij, J.J.; Jalili, A.; van Lieshout, L.; Choi, M.H.; Bae, Y.M.; Lim, M.K.; Hong, S.T. Detection of Clonorchis sinensis in stool samples using real-time PCR. Ann. Trop. Med. Parasitol. 2009, 103, 513-518. [CrossRef] [PubMed]

18. Basuni, M.; Rahumatullah, A.; Miswan, N.; Ahmad, M.; Noordin, R.; Verweij, J.J.; Zainudin, N.S.; Aziz, F.A.; Othman, N.; Muhi, J. A Pentaplex Real-Time Polymerase Chain Reaction Assay for Detection of Four Species of Soil-Transmitted Helminths. Am. J. Trop. Med. Hyg. 2011, 84, 338-343. [CrossRef] [PubMed]

19. Taniuchi, M.; Verweij, J.J.; Noor, Z.; Sobuz, S.U.; Lieshout, L.V.; Petri, W.A., Jr.; Haque, R.; Houpt, E.R. High throughput multiplex PCR and probe-based detection with Luminex beads for seven intestinal parasites. Am. J. Trop. Med. Hyg. 2011, 84, $332-337$. [CrossRef] [PubMed]

20. Liu, J.; Gratz, J.; Amour, C.; Kibiki, G.; Becker, S.; Janaki, L.; Verweij, J.J.; Taniuchi, M.; Sobuz, S.U.; Haque, R.; et al. A LaboratoryDeveloped TaqMan Array Card for Simultaneous Detection of 19 Enteropathogens. J. Clin. Microbiol. 2013, 51, 472-480. [CrossRef] [PubMed]

21. Mejia, R.; Vicuña, Y.; Vaca, M.; Cooper, P.J.; Chico, M.; Nutman, T.B.; Broncano, N.; Sandoval, C. A Novel, Multi-Parallel, Real-Time Polymerase Chain Reaction Approach for Eight Gastrointestinal Parasites Provides Improved Diagnostic Capabilities to Resource-Limited At-Risk Populations. Am. J. Trop. Med. Hyg. 2013, 88, 1041-1047. [CrossRef]

22. Praet, N.; Verweij, J.J.; Mwape, K.E.; Phiri, I.K.; Muma, J.B.; Zulu, G.; Van Lieshout, L.; Rodriguez-Hidalgo, R.; Benitez-Ortiz, W.; Dorny, P.; et al. Bayesian modelling to estimate the test characteristics of coprology, coproantigen ELISA and a novel real-time PCR for the diagnosis of taeniasis. Trop. Med. Int. Health 2013, 18, 608-614. [CrossRef] [PubMed]

23. Van Mens, S.P.; Aryeetey, Y.; Yazdanbakhsh, M.; Van Lieshout, L.; Boakye, D.; Verweij, J.J. Comparison of real-time PCR and Kato smear microscopy for the detection of hookworm infections in three consecutive faecal samples from schoolchildren in Ghana. Trans. R. Soc. Trop. Med. Hyg. 2013, 107, 269-271. [CrossRef]

24. Soonawala, D.; Boer, M.A.M.D.; Visser, L.; Verweij, J.J.; Ratering, M.; Van Lieshout, L.; Claas, E.C.J.; Godkewitsch, A. Post-Travel Screening of Asymptomatic Long-Term Travelers to the Tropics for Intestinal Parasites Using Molecular Diagnostics. Am. J. Trop. Med. Hyg. 2014, 90, 835-839. [CrossRef] [PubMed]

25. Schunk, M.; Kebede Mekonnen, S.; Wondafrash, B.; Mengele, C.; Fleischmann, E.; Herbinger, K.H.; Verweij, J.J.; Geldmacher, C.; Bretzel, G.; Löscher, T.; et al. Use of Occult Blood Detection Cards for Real-Time PCR-Based Diagnosis of Schis-tosoma Mansoni Infection. PLoS ONE 2015, 10, e0137730. [CrossRef] [PubMed]

26. Llewellyn, S.; Inpankaew, T.; Nery, S.V.; Gray, D.J.; Verweij, J.J.; Clements, A.C.; Gomes, S.J.; Traub, R.; McCarthy, J.S. Ap-plication of a Multiplex Quantitative PCR to Assess Prevalence and Intensity Of Intestinal Parasite Infections in a Controlled Clinical Trial. PLoS Negl. Trop. Dis. 2016, 10, e0004380. [CrossRef] [PubMed]

27. Cunningham, L.; Stothard, J.R.; Osei-Atweneboana, M.; Armoo, S.; Verweij, J.J.; Adams, E.R. Developing a real-time PCR assay based on multiplex high-resolution melt-curve analysis: A pilot study in detection and discrimination of soil-transmitted helminth and schistosome species. Parasitology 2018, 145, 1733-1738. [CrossRef] [PubMed]

28. Köller, T.; Hahn, A.; Altangerel, E.; Verweij, J.J.; Landt, O.; Kann, S.; Dekker, D.; May, J.; Loderstädt, U.; Podbielski, A.; et al. Comparison of commercial and in-house real-time PCR platforms for 15 parasites and microsporidia in human stool samples without a gold standard. Acta Trop. 2020, 207, 105516.

29. Poon, R.W.; Tam, E.W.; Lau, S.K.; Cheng, V.C.; Yuen, K.-Y.; Schuster, R.K.; Woo, P.C. Molecular identification of cestodes and nematodes by cox1 gene real-time PCR and sequencing. Diagn. Microbiol. Infect. Dis. 2017, 89, 185-190. [CrossRef] 
30. Wong, S.S.Y.; Poon, R.W.S.; To, K.K.W.; Chan, J.F.-W.; Lu, G.; Xing, F.; Cheng, V.C.C.; Yuen, K.-Y. Improving the specific diagnosis of trematode, cestode and nematode infections by a multiplex single-tube real-time PCR assay. J. Clin. Pathol. 2019, 72, 487-492. [CrossRef]

31. Utzinger, J.; Botero-Kleiven, S.; Castelli, F.; Chiodini, P.L.; Edwards, H.; Köhler, N.; Gulletta, M.; Lebbad, M.; Manser, M.; Matthys, B.; et al. Microscopic diagnosis of sodium acetate-acetic ac-id-formalin-fixed stool samples for helminths and intestinal protozoa: A comparison among European reference laboratories. Clin. Microbiol. Infect. 2010, 16, 267-273. [CrossRef] [PubMed]

32. Moundounga, H.K.; Adegnika, A.A.; Nkoma, A.-M.; Ateba-Ngoa, U.; Mbong, M.; Zinsou, J.; Lell, B.; Verweij, J.J. Impact of Short-Time Urine Freezing on the Sensitivity of an Established Schistosoma Real-Time PCR Assay. Am. J. Trop. Med. Hyg. 2014, 90, 1153-1155. [CrossRef]

33. Kaisar, M.M.M.; Brienen, E.A.T.; Djuardi, Y.; Sartono, E.; Yazdanbakhsh, M.; Verweij, J.J.; Supali, T.; Van Lieshout, L. Improved diagnosis of Trichuris trichiura by using a bead-beating procedure on ethanol preserved stool samples prior to DNA isolation and the performance of multiplex real-time PCR for intestinal parasites. Parasitology 2017, 144, 965-974. [CrossRef] [PubMed]

34. Ayana, M.; Cools, P.; Mekonnen, Z.; Biruksew, A.; Dana, D.; Rashwan, N.; Prichard, R.; Vlaminck, J.; Verweij, J.J.; Levecke, B. Comparison of four DNA extraction and three preservation protocols for the molecular detection and quantification of soiltransmitted helminths in stool. PLOS Negl. Trop. Dis. 2019, 13, e0007778. [CrossRef]

35. Marti, H.; Escher, E. [SAF-an alternative fixation solution for parasitological stool specimens]. Schweiz. Med. Wochenschr. 1990, 120, 1473-1476. [PubMed]

36. Nazer, H.; Greer, W.; Donnelly, K.; E Mohamed, A.; Yaish, H.; Kagalwalla, A.; Pavillard, R. The need for three stool specimens in routine laboratory examinations for intestinal parasites. Br. J. Clin. Pract. 1993, 47, 76-78. [PubMed]

37. Hiatt, R.A.; Markell, E.K.; Ng, E. How Many Stool Examinations are Necessary to Detect Pathogenic Intestinal Protozoa? Am. J. Trop. Med. Hyg. 1995, 53, 36-39. [CrossRef]

38. Baermann, G. Eine einfache Methode zur Auffindung von Ankylostomum-(Nematoden)-Larven in Erdproben. Tijdschr Diergeneeskd Ned-Indie 1917, 57, 131-137.

39. Buonfrate, D.; Formenti, F.; Perandin, F.; Bisoffi, Z. Novel approaches to the diagnosis of Strongyloides stercoralis infection. Clin. Microbiol. Infect. 2015, 21, 543-552. [CrossRef] [PubMed]

40. Cools, P.; van Lieshout, L.; Koelewijn, R.; Addiss, D.; Ajjampur, S.S.R.; Ayana, M.; Bradbury, R.S.; Cantera, J.L.; Dana, D.; Fischer, K.; et al. First interna-tional external quality assessment scheme of nucleic acid amplification tests for the detection of Schistosoma and soil-transmitted helminths, including Strongyloides: A pilot study. PLoS Negl. Trop. Dis. 2020, 14, e0008231. [CrossRef]

41. Wichmann, D.; Panning, M.; Quack, T.; Kramme, S.; Burchard, G.-D.; Grevelding, C.; Drosten, C. Diagnosing Schistosomiasis by Detection of Cell-Free Parasite DNA in Human Plasma. PLoS Negl. Trop. Dis. 2009, 3, e422. [CrossRef]

42. Cnops, L.; Soentjens, P.; Clerinx, J.; Van Esbroeck, M. A Schistosoma haematobium-Specific Real-Time PCR for Diagnosis of Urogenital Schistosomiasis in Serum Samples of International Travelers and Migrants. PLoS Negl. Trop. Dis. 2013, 7, e2413. [CrossRef] [PubMed]

43. Wiemer, D.; Loderstaedt, U.; Von Wulffen, H.; Priesnitz, S.; Fischer, M.; Tannich, E.; Hagen, R.M. Real-time multiplex PCR for simultaneous detection of Campylobacter jejuni, Salmonella, Shigella and Yersinia species in fecal samples. Int. J. Med Microbiol. 2011, 301, 577-584. [CrossRef] [PubMed]

44. Landis, J.R.; Koch, G.G. The Measurement of Observer Agreement for Categorical Data. Biomedicine 1977, 33, 159. [CrossRef]

45. Sedgwick, P. Multiple hypothesis testing and Bonferroni's correction. BMJ 2014, 349, g6284. [CrossRef] [PubMed]

46. Rådström, P.; Knutsson, R.; Wolffs, P.; Lövenklev, M.; Löfström, C. Pre-PCR processing: Strategies to generate PCR-compatible samples. Mol. Biotechnol. 2004, 26, 133-146. [CrossRef]

47. Niesters, H.G. Quantitation of Viral Load Using Real-Time Amplification Techniques. Methods 2001, 25, 419-429. [CrossRef] [PubMed] 